\title{
Threshold phenomena for symmetric decreasing solutions of reaction-diffusion equations
}

\author{
C. B. Muratov and X. Zhong
}

\begin{abstract}
We study the long time behavior of solutions of the Cauchy problem for nonlinear reaction-diffusion equations in one space dimension with the nonlinearity of bistable, ignition or monostable type. We prove a one-to-one relation between the long time behavior of the solution and the limit value of its energy for symmetric decreasing initial data in $L^{2}$ under minimal assumptions on the nonlinearities. The obtained relation allows to establish sharp threshold results between propagation and extinction for monotone families of initial data in the considered general setting.
\end{abstract}

Mathematics Subject Classification (2010). 35K57, 35K15, 35A15.

Keywords. Sharp transition; traveling waves; gradient flow.

\section{Introduction}

In this paper, we study the Cauchy problem for the nonlinear reaction-diffusion equation

$$
\begin{aligned}
& u_{t}=u_{x x}+f(u), \quad x \in \mathbb{R}, t>0, \\
& u(x, 0)=\phi(x) \geq 0 \quad x \in \mathbb{R}, \quad \phi \in L^{2}(\mathbb{R}) \cap L^{\infty}(\mathbb{R}) .
\end{aligned}
$$

The nonlinearity $f$ satisfies

$$
f \in C^{1}([0, \infty)), \quad f(0)=f(1)=0, \quad f(u)<0 \quad \text { for } \quad u>1 .
$$

We are interested in the long time behavior of solution of (1.1). Since $u=0$ and $u=1$ are solutions of the stationary problem for (1.1), one possible behavior of the solution is extinction, i.e., $\lim _{t \rightarrow \infty} u(x, t)=0$ uniformly in $\mathbb{R}$. Another

This work was supported by NSF via grants DMS-0718027, DMS-0908279 and DMS1119724 . 
possible behavior of the solution is propagation, i.e., $\lim _{t \rightarrow \infty} u(x, t)=1$ locally uniformly in $\mathbb{R}$ and, moreover, $\lim _{t \rightarrow \infty} u(x+c t, t)=1$ locally uniformly for all sufficiently small $c \in \mathbb{R}$. This type of question was first posed in the context of combustion modeling, where the considered initial value problem prominently appears [1-3], and is also relevant to numerous other applications in physics, chemistry and biology (see, e.g., [4-7]). In the context of combustion, when cold fuel and oxidizer gases are premixed in a tube, a sufficiently large region of heated gas generated, say, by a spark will ignite a pair of counter-propagating flame fronts, while insufficient heating will fail to result in ignition. Understanding the nature of the threshold phenomena associated with ignition is, therefore, important for many phenomena governed by reaction and diffusion processes.

Mathematical studies of the ignition problem date back to the early 1960s. In his pioneering work, Kanel' [8] considered the long time behavior of solution of (1.1) with ignition nonlinearity $f$, whose initial condition $\phi$ is the characteristic function $\chi_{[-L, L]}(x)$ of the interval $[-L, L]$. He proved that there exist constants $L_{1} \geq L_{0}>0$, depending on $f$, such that extinction occurs when $L<L_{0}$, and propagation occurs when $L>L_{1}$. Aronson and Weinberger [9] extended this result to bistable nonlinearities and more general initial conditions. These works, however, did not provide any further information on the nature of the transition between ignition and extinction.

Further insight into the ignition problem was provided very recently by Zlatoš [10] (see also related works $[11,12]$ ), who proved that in the problem studied by Kanel' it is possible to choose $L_{0}=L_{1}$, i.e., the transition from extinction to propagation is sharp. He also found that the long time behavior of the solution with the initial data corresponding to the threshold value $L_{0}$ is neither extinction nor propagation. In particular, for bistable nonlinearities the solution of the initial value problem with the data corresponding to $L_{0}$ converges to the stationary "bump" solution of (1.1), i.e., the unique symmetric decreasing solution of

$$
v^{\prime \prime}(x)+f(v(x))=0, \quad x \in \mathbb{R} .
$$

Du and Matano [13] generalized the sharp transition result of Zlatoš to monotone families of compactly supported initial data by using the zero number counting argument. By a different method, Poláčik [14] gave a higher-dimensional extension, still for compactly supported initial data.

As was pointed out by Matano [15], for bistable nonlinearities all the works on sharp threshold behavior between ignition and extinction mentioned above crucially rely on the assumption of the data being compactly supported (or rapidly decaying) and, therefore, may not be applied to data that lie in the natural function spaces, such as, e.g., $L^{2}(\mathbb{R})$. The purpose of this work is to provide such an extension in the context of the problem originally considered by Kanel'. To achieve this goal, we take advantage of the gradient flow structure of the considered equation and develop energy-based methods that are quite different from those used in the above works (for some related works, see [16-18]). One of the main tools for our analysis of the threshold behavior 
is the result on a one-to-one correspondence between the long time behavior of the solution and that of its suitably defined energy that we establish in this paper.

As in the work of Du and Matano [13], we consider an increasing oneparameter family of initial conditions $\phi_{\lambda}, \lambda>0$, satisfying conditions in (1.2), with $\lim _{\lambda \rightarrow 0} \phi_{\lambda} \equiv 0$, and the map $\lambda \mapsto \phi_{\lambda}$ increasing and continuous in the $L^{2}(\mathbb{R})$ norm. We also require an additional technical assumption that $\phi_{\lambda}(x)$ be a symmetric decreasing function of $x$ :

(SD) The initial condition $\phi(x)$ in (1.2) is symmetric decreasing, i.e., $\phi(-x)=$ $\phi(x)$ and $\phi(x)$ is non-increasing for every $x>0$.

This assumption allows us to avoid a possible long-time behavior consisting of a bump solution slowly moving off to infinity, which was pointed out for some related problems [19]. We note that assumption (SD) is equivalent to considering (1.1) on half-line with Neumann boundary condition and non-increasing initial data (for some related results, see [17]).

In the case of bistable and ignition nonlinearities (for precise definitions and statements, see the following section) it is easy to show that if the parameter $\lambda$ is small enough, then extinction occurs. We then wish to know if propagation can occur when $\lambda$ is large. And a more interesting question is: does there exist any long time behavior of solution, which is neither extinction, nor propagation, for intermediate values of $\lambda$ ? On the other hand, for monostable nonlinearities it is known that propagation occurs for any $\lambda>0$ if $f^{\prime}(0)>0$ [9], or even when $f(u) \sim u^{p}$ for small $u$, when $p \leq p_{c}$, where $p_{c}=3$ is the Fujita exponent in one space dimension (see e.g., [20,21]). Nevertheless, the question of long-time behavior is also non-trivial for $p>p_{c}$ and to the best of our knowledge has not been treated so far.

Here we prove, for bistable and ignition nonlinearities, that if propagation occurs at some value of $\lambda>0$, then there is a value of $\lambda=\lambda^{*}>0$ which serves as a sharp threshold between propagation for $\lambda>\lambda^{*}$ and extinction for $\lambda<\lambda^{*}$. We also characterize the behavior of solution at $\lambda=\lambda^{*}$, thus generalizing the result of Zlatoš to the considered class of data. And for monostable nonlinearities which are supercritical with respect to the Fujita exponent, we prove that if propagation occurs at some value of $\lambda>0$, then there exists a value $\lambda^{*}>0$, which serves as a sharp threshold between propagation for $\lambda>\lambda^{*}$ and extinction at $\lambda \leq \lambda^{*}$. Note that in this case propagation and extinction exhaust the list of possible long-time behaviors of solutions. In addition, we obtain a new sufficient condition for propagation which can be easily verified. We also note that with minor modifications many of our conclusions still hold if $f(u)$ is only locally Lipschitz.

Our paper is organized as follows. In Sect. 2 we introduce the background results related to the variational structure of the considered problem. Then in Sect. 3 we consider bistable nonlinearities and give our convergence result in Theorem 1, our one-to-one relation result in Theorem 2 and our sharp threshold result in Theorem 3. Then in Sect. 4 we treat monostable nonlinearities and give our convergence result in Theorem 4, our one-to-one relation result 
in Theorem 5 and our sharp threshold result in Theorem 6, and in Sect. 5 we present results for ignition nonlinearities, with our convergence result in Theorem 7, the relation with the limit energy in Theorem 8 and our sharp threshold result in Theorem 9 .

\section{Preliminaries}

We first recall that existence of classical solutions for (1.1) with initial data satisfying (1.2) is well known. In view of (1.3), these solutions are positive, uniformly bounded and, hence, global in time. Furthermore, it is well know that the derivatives $u_{t}(x, t), u_{x}(x, t), u_{x x}(x, t)$ of the solution of (1.1) can be estimated in the uniform norm in terms of $u$ itself. More precisely, the uniform boundedness of $|u|$ in the half-space $t>0$ controls the boundedness of $\left|u_{t}\right|$, $\left|u_{x}\right|$ and $\left|u_{x x}\right|$ in the half-space $t \geq T$ for any $T>0$ (see, e.g., [22,23]). We will refer to this boundedness as "standard parabolic regularity." For our purposes here, however, we will also need a suitable existence theory for solutions in integral norms that measure, in some sense, the rate of the decay of solutions as $x \rightarrow \pm \infty$. This is because we wish to work with the energy functional, defined as

$$
E[u]:=\int_{\mathbb{R}}\left(\frac{1}{2} u_{x}^{2}+V(u)\right) d x, \quad V(u):=-\int_{0}^{u} f(s) d s .
$$

Clearly, this functional is well-defined for any $u \in H^{1}(\mathbb{R}) \cap L^{\infty}(\mathbb{R})$ and of class $C^{1}$ in $H^{1}(\mathbb{R})$. Similarly, for a given $c>0$ we define the exponentially weighted functional $\Phi_{c}$ associated with (2.1) as

$$
\Phi_{c}[u]:=\int_{\mathbb{R}} e^{c x}\left(\frac{1}{2} u_{x}^{2}+V(u)\right) d x,
$$

which is well-defined for $L^{\infty}$ functions in the exponentially weighted Sobolev space $H_{c}^{1}(\mathbb{R})$ with the norm

$$
\|u\|_{H_{c}^{1}}^{2}:=\|u\|_{L_{c}^{2}}^{2}+\left\|u_{x}\right\|_{L_{c}^{2}}^{2}, \quad\|u\|_{L_{c}^{2}}^{2}:=\int_{\mathbb{R}} e^{c x} u^{2} d x .
$$

Similarly, we can define the space $H_{c}^{2}(\mathbb{R})$ as the space of functions whose first derivatives belong to $H_{c}^{1}(\mathbb{R})$.

The following proposition guarantees existence and regularity properties of solutions of (1.1) in both the usual and the exponentially weighted Sobolev spaces.

Proposition 2.1. Under (1.3), there exists a unique solution $u \in C_{1}^{2}(\mathbb{R} \times(0, \infty))$ $\left.\cap L^{\infty}(\mathbb{R} \times(0, \infty))\right)$ satisfying (1.1) and (1.2) (using the notations from [24]), with

$$
u \in C\left([0, \infty) ; L^{2}(\mathbb{R})\right) \cap C\left((0, \infty) ; H^{2}(\mathbb{R})\right)
$$

and $u_{t} \in C\left((0, \infty) ; H^{1}(\mathbb{R})\right)$. Furthermore, if there exists $c>0$ such that the initial condition $\phi(x) \in L_{c}^{2}(\mathbb{R}) \cap L^{\infty}(\mathbb{R})$, then the solution of (1.1) and (1.2) 
satisfies

$$
u \in C\left([0, \infty) ; L_{c}^{2}(\mathbb{R})\right) \cap C\left((0, \infty) ; H_{c}^{2}(\mathbb{R})\right),
$$

with $u_{t} \in C\left((0, \infty) ; H_{c}^{1}(\mathbb{R})\right)$. In addition, small variations of the initial data in $L^{2}(\mathbb{R})$ result in small changes of solution in $H^{1}(\mathbb{R})$ at any $t>0$.

Proof. Follows from the arguments in the proof of [25, Proposition 3.1] based on the approach of [26], taking into consideration that by (1.3) the function $\bar{u}(x, t)=\max \left\{1,\|\phi\|_{L^{\infty}(\mathbb{R})}\right\}$ is a universal supersolution for the considered problem.

Remark 2.2. We note that Proposition 2.1 does not require hypothesis (SD). However, under (SD) we also have that $u(x, t)$ is a symmetric decreasing function of $x$ for all $t>0$.

In view of Proposition 2.1, by direct calculation we obtain the well-known identity related to the energy dissipation rate for the solutions of (1.1) valid for all $t>0$ :

$$
\frac{d E}{d t}[u(\cdot, t)]=-\int_{\mathbb{R}} u_{t}^{2}(x, t) d x .
$$

In fact, the basic reason for (2.4) is the fact that (1.1) is a gradient flow in $L^{2}$ generated by E. Similarly, as was first pointed out in [27], Eq. (1.1) written in the reference frame moving with an arbitrary speed $c>0$ is a gradient flow in $L_{c}^{2}$ generated by $\Phi_{c}$. More precisely, defining $\tilde{u}(x, t):=u(x+c t, t)$, which solves

$$
\tilde{u}_{t}=\tilde{u}_{x x}+c \tilde{u}_{x}+f(\tilde{u}),
$$

it is easy to see with the help of Proposition 2.1 that an identity similar to (2.4) holds for $\Phi_{c}$ :

$$
\frac{d \Phi_{c}}{d t}[\tilde{u}(\cdot, t)]=-\int_{\mathbb{R}} e^{c x} \tilde{u}_{t}^{2}(x, t) d x .
$$

In particular, both $E[u(\cdot, t)]$ and $\Phi_{c}[\tilde{u}(\cdot, t)]$ are well defined and are non-increasing in $t$ for all $t>0$. Also note that non-trivial fixed points of (2.5) are variational traveling waves, i.e., solutions that propagate with constant speed $c>0$ invading the equilibrium $u=0$ and belong to $H_{c}^{1}(\mathbb{R})$ [28]. Furthermore, as was shown in [28], for sufficiently rapidly decaying front-like initial data the propagation speed associated with the leading edge of the solution (see the next paragraph for the definition) is determined by the special variational traveling wave solutions which are minimizers of $\Phi_{c}$ for some unique speed $c=c^{\dagger}>0$. In the context of the nonlinearities considered in this paper, the following proposition gives existence, uniqueness and several properties of these minimizers (follows directly from [28, Theorem 3.3]; in fact, under these assumptions they are the only variational traveling waves, see [25, Corollary 3.4]).

Proposition 2.3. Let $f$ satisfy (1.3), let $f^{\prime}(0) \leq 0$, and let $u_{0}=1$ be the unique zero of $f$ such that $\int_{0}^{u_{0}} f(u) d u>0$. Then there exists a unique $c^{\dagger}>0$ and $a$ unique (up to translation) positive traveling wave solution $u(x, t)=\bar{u}\left(x-c^{\dagger} t\right)$ 
of $(1.1)$ such that $\bar{u}(+\infty)=0, \bar{u}(-\infty)=1, \bar{u}^{\prime}<0$, and $\bar{u}$ minimizes $\Phi_{c}$ with $c=c^{\dagger}$.

Turning back to the question of propagation, for a given $\delta>0$ we define the leading edge $R_{\delta}(t)$ of the solution $u(x, t)$ of (1.1) as

$$
R_{\delta}(t):=\sup \{x \in \mathbb{R}: u(x, t) \geq \delta\} .
$$

If the set $\{x \in \mathbb{R}: u(x, t) \geq \delta\}=\varnothing$, then $R_{\delta}(t):=-\infty$. Then, as follows from [28, Theorem 5.8], under the assumptions of Proposition 2.3 for every $\phi \in L_{c}^{2}(\mathbb{R})$ with some $c>c^{\dagger}, \phi(x) \in[0,1]$ for all $x \in \mathbb{R}$, and $\lim _{x \rightarrow-\infty} \phi(x)=1$ the leading edge $R_{\delta}(t)$ propagates asymptotically with speed $c^{\dagger}$ for sufficiently small $\delta>0$. Similarly, the same conclusion holds for the initial data obeying (1.2), provided that $\phi \in L_{c}^{2}(\mathbb{R})$ with some $c>c^{\dagger}$ and $u(x, t) \rightarrow 1$ as $t \rightarrow \infty$ locally uniformly in $x \in \mathbb{R}[28$, Corollary 5.9]. In fact, a stronger conclusion can be made, which implies that the latter condition is equivalent to the stronger notion of propagation presented in the introduction, extending the results of Aronson and Weinberger [9, Theorem 4.5] to the considered class of nonlinearities.

Proposition 2.4. Under the assumptions of Proposition 2.3, let $\phi$ satisfy (1.2) and assume that $u(x, t) \rightarrow 1$ as $t \rightarrow \infty$ locally uniformly in $x \in \mathbb{R}$. Then for every $\delta_{0} \in(0,1)$ and every $c \in\left(0, c^{\dagger}\right)$, where $c^{\dagger}$ is the same as in Proposition 2.3, there exists $T \geq 0$ such that $R_{\delta}(t) \geq$ ct for every $t \geq T$ and every $\delta \in\left(0, \delta_{0}\right]$.

Proof. Consider minimizers of $\Phi_{c}$ among $u \in X$, where $X$ consists of all functions in $H_{c}^{1}(\mathbb{R})$ with values in $[0,1]$ that vanish for all $x>0$. We claim that a non-trivial minimizer $\bar{u}_{c} \in X$ of $\Phi_{c}$ exists for all $c \in\left(0, c^{\dagger}\right)$. Indeed, by the argument in the proof of [28, Proposition 5.5], we have $\inf _{u \in X} \Phi_{c}[u]<0$ for any $c \in\left(0, c^{\dagger}\right)$. By boundedness of $u \in X, \Phi_{c}$ is coercive on $X$. Existence of a minimizer then follows from weak sequential lower semicontinuity of $\Phi_{c}$ on $X$ (see [29, Lemma 5.3]). Furthermore, by [29, Corollary 6.8], which can be easily seen to be applicable to $\bar{u}_{c}$, we have $\bar{u}_{c}(x) \rightarrow 1$ as $x \rightarrow-\infty$.

Similarly, for large enough $R>0$ there exists a non-trivial minimizer $\bar{u}_{c}^{R} \in X_{R}$ of $\Phi_{c}$, where $X_{R}$ is a subset of $X$ with all functions vanishing for $x<-R$ as well. These are stationary solutions of $(2.5)$ with Dirichlet boundary conditions at $x=0$ and $x=-R$, and by strong maximum principle we have $\bar{u}_{c}^{R}<1$. Furthermore, if $R_{n} \rightarrow \infty$, then $\left\{\bar{u}_{c}^{R_{n}}\right\}$ constitute a minimizing sequence for $\Phi_{c}$ in $X$ and, in view of the continuity of $\int_{-\infty}^{0} e^{c x} V(u) d x$ with respect to the weak convergence in $H_{c}^{1}(\mathbb{R})$ we have $\bar{u}_{c}^{R_{n}} \rightarrow \bar{u}_{c}$ strongly in $H_{c}^{1}(\mathbb{R})$ and, by Sobolev imbedding, also locally uniformly. In particular, $\left\|\bar{u}_{c}^{R_{n}}\right\|_{L^{\infty}(\mathbb{R})} \rightarrow 1$ as $n \rightarrow \infty$. The proof is then completed by using $\bar{u}_{c}^{R_{n}}$ with a large enough $n$ depending on $\delta_{0}$ as a subsolution after a sufficiently long time $t$.

Remark 2.5. If in Proposition 2.4 we also have $\phi \in L_{c}^{2}(\mathbb{R})$ for some $c>c^{\dagger}$, then by [28, Proposition 5.2] for every $\delta_{0}>0$ and every $c^{\prime}>c^{\dagger}$ there exists $T \geq 0$ such that $R_{\delta}(t)<c^{\prime} t$ for every $\delta \geq \delta_{0}$, implying that $c^{\dagger}$ is the sharp 
propagation velocity for the level sets in the above sense. The same conclusion also holds for the "trailing edge", i.e., the leading edge defined using $u(-x, t)$ instead of $u(x, t)$, indicating the formation of a pair of counter-propagating fronts with speed $c^{\dagger}$.

Remark 2.6. Under hypothesis (SD), the conclusion of Proposition 2.4 clearly implies propagation in the sense defined in the introduction.

The difficult part in applying Proposition 2.4 is to establish that $u(x, t) \rightarrow$ 1 locally uniformly in $x \in \mathbb{R}$ as $t \rightarrow \infty$ for a given initial condition $\phi(x)$. In the absence of such a result, we can still appeal to a weaker notion of propagation of the leading edge analyzed in [27]. Following [27], we call the solution $u(x, t)$ of (1.1) and (1.2) wave-like, if there exist constants $c>0$ and $T \geq 0$ such that $\phi \in L_{c}^{2}(\mathbb{R})$ and $\Phi_{c}[u(\cdot, T)]<0$. Note that by monotonicity of $\Phi_{c}[\tilde{u}(\cdot, t)]$ and the fact that $\Phi_{c}[u(\cdot, t)]=e^{c^{2} t} \Phi_{c}[\tilde{u}(\cdot, t)]$, it follows that for a wave-like solution we have $\Phi_{c}[u(\cdot, t)]<0$ for all $t \geq T$ as well. This fact allows to obtain an important characterization of the leading edge dynamics for wave-like solutions which is intimately related to the gradient descent structure of (2.5). We note that in view of the "hair-trigger effect" discussed in the introduction in the case when $u=0$ is linearly unstable [9], we only need to consider the nonlinearities satisfying $f^{\prime}(0) \leq 0$.

Proposition 2.7. Let $f$ satisfy $(1.3)$, let $f^{\prime}(0) \leq 0$, and let $u(x, t)$ be a wave-like solution of (1.1) and (1.2), i.e., $\Phi_{c}[u(\cdot, T)]<0$ for some $c>0$ and $T \geq 0$. Then for all $t \geq T$

$$
\max _{x \in \mathbb{R}} u(x, t) \geq \delta_{0}
$$

where

$$
\delta_{0}=\inf \left\{u \geq 0: V(u)<-\frac{1}{8} c^{2} u^{2}\right\}>0 .
$$

Furthermore, there exists $R_{0} \in \mathbb{R}$ such that for every $\delta \in\left(0, \delta_{0}\right]$ we have

$$
R_{\delta}(t) \geq c t+R_{0}
$$

for all $t \geq T$.

Proof. The statement is a direct consequence of [27, Proposition 4.10 and Theorem 4.11], which remain valid under the assumptions above in view of Proposition 2.1. For the reader's convenience, we outline the proof here.

By the definition of a wave-like solution, $\Phi_{c}[u(x, T)]<0$ for some $c>0$ and $T \geq 0$. By (2.6), we know that for all $t \geq T$,

$$
\Phi_{c}[u(x+c(t-T), t)] \leq \Phi_{c}[u(x, T)]<0 .
$$

Moreover, we have the following obvious property of $\Phi_{c}[29$, Lemma 3.1]

$$
\Phi_{c}[u(x-R, t)]=e^{c R} \Phi_{c}[u(x, t)],
$$

which implies that the sign of $\Phi_{c}$ is invariant with respect to translations [27, Corollary 2.4]. In particular, $\Phi_{c}[u(x, t)]<0$ for all $t \geq T$. So that

$$
\inf _{x \in \mathbb{R}}\left(\frac{1}{2} u_{x}^{2}(x, t)+V(u(x, t))\right)<0, \quad \forall t \geq T \text {. }
$$


Furthermore, by [29, Lemma 2.1] we have

$$
\frac{c^{2}}{4} \int_{\mathbb{R}} e^{c x} u^{2}(x, t) d x \leq \int_{\mathbb{R}} e^{c x} u_{x}^{2}(x, t) d x .
$$

So (2.13) implies (2.8). In addition, for any $c>0$, the hypothesis $f^{\prime}(0) \leq 0$ guarantees that $\delta_{0}>0$.

Let us now prove (2.10). It is enough to verify (2.10) for $\delta=\delta_{0}$, because $R_{\delta}(t) \geq R_{\delta_{0}}(t)$ whenever $\delta<\delta_{0}$. Equation (1.3) implies that $V(u)$ attains its minimum $V_{\text {min }} \leq 0$ for $u \geq 0$. Moreover, by (2.9) we have $V(u(x, t))>0$ for all $x>R_{\delta_{0}}(t)$. Using (2.12), we obtain

$$
\begin{aligned}
\Phi_{c}\left[u\left(x+R_{\delta_{0}}(t), t\right)\right] & =e^{-c R_{\delta_{0}}(t)} \Phi_{c}\left[u\left(x+R_{\delta_{0}}(t), t\right)\right] \\
& =\int_{\mathbb{R}} e^{c\left(x-R_{\delta_{0}}(t)\right)}\left(\frac{1}{2} u_{x}^{2}(x, t)+V(u(x, t))\right) d x \\
& \geq \int_{-\infty}^{R_{\delta_{0}}(t)} e^{c\left(x-R_{\delta_{0}}(t)\right)} V_{\min } d x \\
& =\frac{V_{\text {min }}}{c} .
\end{aligned}
$$

Then, using (2.11), (2.12), and (2.15) together, we have

$$
\begin{aligned}
0>e^{-c\left(R_{\delta_{0}}(t)-c t\right)} \Phi_{c}[u(x+c T, T)] & \geq e^{-c\left(R_{\delta_{0}}(t)-c t\right)} \Phi_{c}[u(x+c t, t)] \\
& =\Phi_{c}\left[u\left(x+R_{\delta_{0}}(t), t\right)\right] \\
& \geq \frac{V_{m i n}}{c} .
\end{aligned}
$$

Dividing $(2.16)$ by a negative number $\Phi_{c}[u(x+c T, T)]$ and taking the logarithm of both sides, we have

$$
R_{\delta_{0}}(t) \geq c t+\frac{1}{c} \ln \frac{c \Phi_{c}[u(x+c T, T)]}{V_{\min }},
$$

which implies (2.10).

One of the goals of our analysis in the following sections will be to show that under further assumptions on the nonlinearities and hypothesis (SD) propagation in the sense of Proposition 2.7 implies propagation in the sense of Proposition 2.4. We note, however, that having merely $E[u(\cdot, T)]<0$ for some $T \geq 0$ is not yet sufficient for employing Proposition 2.7 , since we cannot assume a priori that $u(x, t)$ decays exponentially as $|x| \rightarrow \infty$. We will overcome the difficulty in the next section.

A key ingredient of our proofs that allows us to efficiently use variational methods and to go from sequential limits to full limits as $t \rightarrow \infty$ without much information about the limit states relies on an interesting observation regarding uniform Hölder continuity of the solutions of (1.1) with bounded energy. This result is stated in the following proposition. We note that a more general result is also available in $\mathbb{R}^{N}$ (it will be discussed in more detail elsewhere).

Proposition 2.8. Suppose that $\phi$ satisfies (1.2) and $f$ satisfies $(1.3)$. If $E[u(\cdot, t)]$ is bounded from below, then $u(x, \cdot) \in C^{1 / 4}([T, \infty))$ for each $x \in \mathbb{R}$ and each 
$T>0$. Moreover, the corresponding Hölder constant of $u(x, t)$ converges to 0 as $T \rightarrow \infty$ uniformly in $x$.

Proof. We denote $E_{\infty}=\lim _{t \rightarrow \infty} E[u(\cdot, t)]$. Then, using (2.4), for any $x_{0} \in \mathbb{R}$ and $t_{2}>t_{1} \geq T$ we have

$$
\begin{aligned}
\int_{x_{0}}^{x_{0}+1}\left|u\left(x, t_{2}\right)-u\left(x, t_{1}\right)\right| d x & \leq \int_{t_{1}}^{t_{2}} \int_{x_{0}}^{x_{0}+1}\left|u_{t}(x, t)\right| d x d t \\
& \leq \sqrt{t_{2}-t_{1}}\left(\int_{t_{1}}^{t_{2}} \int_{x_{0}}^{x_{0}+1} u_{t}^{2}(x, t) d x d t\right)^{1 / 2} \\
& \leq \sqrt{t_{2}-t_{1}}\left(\int_{T}^{\infty} \int_{\mathbb{R}} u_{t}^{2}(x, t) d x d t\right)^{1 / 2} \\
& =\sqrt{\left(E[u(\cdot, T)]-E_{\infty}\right)\left(t_{2}-t_{1}\right)} .
\end{aligned}
$$

On the other hand, by standard parabolic regularity there exists $M>0$ such that

$$
\left\|u_{x}(\cdot, t)\right\|_{L^{\infty}(\mathbb{R})} \leq M, \quad\|u(\cdot, t)\|_{L^{\infty}(\mathbb{R})} \leq M \quad \forall t \geq T,
$$

Without loss of generality we can further assume that $u\left(x_{0}, t_{2}\right)-u\left(x_{0}, t_{1}\right) \in$ $[0, M]$. Then, for every $x \in I$, where

$$
I:=\left[x_{0}, x_{0}+\frac{u\left(x_{0}, t_{2}\right)-u\left(x_{0}, t_{1}\right)}{2 M}\right], \quad|I|<1,
$$

we have

$$
\begin{aligned}
u\left(x, t_{2}\right) & \geq u\left(x_{0}, t_{2}\right)-M\left(x-x_{0}\right) \geq u\left(x_{0}, t_{1}\right)+M\left(x-x_{0}\right) \\
& \geq u\left(x, t_{1}\right), x \in I .
\end{aligned}
$$

This implies that

$$
\begin{aligned}
\int_{x_{0}}^{x_{0}+1}\left|u\left(x, t_{2}\right)-u\left(x, t_{1}\right)\right| d x & \geq \int_{I}\left(u\left(x_{0}, t_{2}\right)-u\left(x_{0}, t_{1}\right)-2 M\left(x-x_{0}\right)\right) d x \\
& =\frac{\left|u\left(x_{0}, t_{2}\right)-u\left(x_{0}, t_{1}\right)\right|^{2}}{4 M}
\end{aligned}
$$

Then we have

$$
\left|u\left(x_{0}, t_{2}\right)-u\left(x_{0}, t_{1}\right)\right| \leq 2 \sqrt{M}\left(E[u(\cdot, T)]-E_{\infty}\right)^{1 / 4}\left(t_{2}-t_{1}\right)^{1 / 4},
$$

i.e., $u(x, \cdot) \in C^{1 / 4}([T, \infty))$ by the arbitrariness of $x_{0}$. Moreover, the limit of the Hölder constant is

$$
\lim _{T \rightarrow \infty} 2 \sqrt{M}\left(E[u(\cdot, T)]-E_{\infty}\right)^{1 / 4}=0,
$$

which completes the proof. 


\section{Bistable Nonlinearity}

We now turn our attention to the study of the bistable nonlinearity, i.e., $f \in$ $C^{1}([0, \infty) ; \mathbb{R})$

$$
f(0)=f\left(\theta_{0}\right)=f(1)=0, \quad f(u) \begin{cases}<0, & \text { in }\left(0, \theta_{0}\right) \cup(1, \infty), \\ >0, & \text { in }\left(\theta_{0}, 1\right),\end{cases}
$$

for some $\theta_{0} \in(0,1)$. In the following, we assume an extra condition that the $u=$ 1 equilibrium is more energetically favorable than the $u=0$ equilibrium, i.e.,

$$
V(1)=-\int_{0}^{1} f(s) d s<0 .
$$

Actually, in the context of threshold phenomena this is not a restriction, since propagation (in the sense defined in the introduction) becomes impossible in the opposite case. Indeed, if the inequality opposite to (3.2) holds, then we have $V(u) \geq 0$ for all $u \geq 0$ and, therefore, $R_{\delta} \leq c t$ for any $\delta>0$, any $c>0$ and large enough $t$, at least for all $\phi \in L_{c}^{2}(\mathbb{R})$ by $[28$, Proposition 5.2]. Furthermore, if $V(1)>0$ and $f^{\prime}(0)<0$ (the latter condition is not essential and may be replaced by a weaker non-degeneracy condition introduced in the next paragraph), then the energy functional in (2.1) is coercive in $H^{1}(\mathbb{R})$, and so it is not difficult to see that every solution of (1.1) and (1.2) converges uniformly to zero, implying extinction for all initial data. Thus the only case in which the situation may be subtle is that of a balanced bistable nonlinearity, i.e., when $V(1)=0$, in which spreading, i.e., sublinear behavior of the leading edge with time, namely $R_{\delta}(t) \rightarrow \infty$ as $t \rightarrow \infty$, but $R_{\delta}(t)=o(t)$ for some $\delta>0$, cannot be excluded a priori, even for exponentially decaying initial data. The analysis of the balanced case is beyond the scope of the present paper.

We further make a kind of weak non-degeneracy assumption that $f(u) \simeq$ $-k u^{p}$ for some $p \geq 1$ and $k>0$ as $u \rightarrow 0$. More precisely, we assume that

$$
f^{\prime}(u) \leq 0 \text { for all } u \in\left[0, \theta_{1}\right], \quad \text { for some } \theta_{1}>0,
$$

and

$$
\lim _{u \rightarrow 0} \frac{f(u)}{u^{p}}=-k \quad \text { for some } p \geq 1 \text { and } \quad k>0 .
$$

Note that (3.3) and (3.4) are automatically satisfied for the generic non-degenerate case when $f^{\prime}(0)<0$. Under conditions (3.1) and (3.2), there exist two roots of $V(u): u=0, u=\theta^{*} \in(0,1)$, and possibly a third root $u=\theta^{\diamond}>1$. However, since by $(1.3)$ we have $\limsup _{t \rightarrow \infty}\|u(x, t)\|_{L^{\infty}(\mathbb{R})} \leq 1$, without loss of generality, in the latter case we may suppose that $\|\phi\|_{L^{\infty}(\mathbb{R})}<\theta^{\diamond}$. This implies that once $u>\theta^{*}$, we have $V(u)<0$.

It is well known that under our assumptions (1.4) possesses "bump" solutions, i.e., classical positive solutions of (1.4) that vanish at infinity. After a suitable translation, these solutions are known to be symmetric decreasing and unique (see, e.g., [30, Theorem 5]). In the following proposition we summarize the properties of the bump solution that are needed for our analysis. 
Proposition 3.1. Let $f$ satisfy conditions (1.3) and (3.1) through (3.4), and let $v \in C^{2}(\mathbb{R})$ be the unique positive symmetric decreasing solution of (1.4). Then

1. $v(0)=\theta^{*}$ and $E_{0}:=E[v]>0$.

2. If $f^{\prime}(0)<0$, we have $v(x), v^{\prime}(x), v^{\prime \prime}(x) \sim e^{-\mu|x|}$ for $\mu=\sqrt{\left|f^{\prime}(0)\right|}$ as $|x| \rightarrow \infty$.

3. If $f^{\prime}(0)=0$, then $v(x) \sim|x|^{-\frac{2}{p-1}}, v^{\prime}(x) \sim|x|^{-\frac{p+1}{p-1}}, v^{\prime \prime}(x) \sim|x|^{-\frac{2 p}{p-1}}$ as $|x| \rightarrow \infty$.

4. $v^{\prime} \in H^{1}(\mathbb{R})$.

Proof. The fact that $v(0)=\theta^{*}$ follows from [30, Theorem 5]. Integrating (1.4) once, we obtain $\left|v^{\prime}\right|=\sqrt{2 V(v)}$, where by the previous result the constant of integration is zero. Upon second integration we arrive at

$$
|x|=\int_{v}^{\theta^{*}} \frac{d u}{\sqrt{2 V(u)}} .
$$

The proof then follows by a careful analysis of the singularity in the integral in (3.5) to establish the decay of the solution. Once the decay is known, the rest of the statements follows straightforwardly.

Our main theorems in this section are about the following convergence and equivalence conclusions.

Theorem 1. Let $f$ satisfy conditions (1.3) and (3.1) through (3.4). Let $\phi(x)$ satisfy condition (1.2) and hypothesis (SD). Then one of the following holds.

1. $\lim _{t \rightarrow \infty} u(x, t)=1$ locally uniformly in $\mathbb{R}$,

2. $\lim _{t \rightarrow \infty} u(x, t)=v(x)$ uniformly in $\mathbb{R}$,

3. $\lim _{t \rightarrow \infty} u(x, t)=0$ uniformly in $\mathbb{R}$.

We will prove Theorem 1 together with establishing the following oneto-one relation between the long time behavior of the solutions and those of their energy $E$.

Theorem 2. Under the same assumptions as in Theorem 1, we have the following three alternatives:

1. $\lim _{t \rightarrow \infty} u(x, t)=1$ locally uniformly in $\mathbb{R} \Leftrightarrow \lim _{t \rightarrow \infty} E[u(\cdot, t)]=-\infty$.

2. $\lim _{t \rightarrow \infty} u(x, t)=v(x)$ uniformly in $\mathbb{R} \Leftrightarrow \lim _{t \rightarrow \infty} E[u(\cdot, t)]=E_{0}$.

3. $\lim _{t \rightarrow \infty} u(x, t)=0$ uniformly in $\mathbb{R} \Leftrightarrow \lim _{t \rightarrow \infty} E[u(\cdot, t)]=0$.

The strategy of our proof is as follows. We wish to show that the limit behaviors of the energy in Theorem 2 are the only possible ones. So we first prove that if $E[u(\cdot, t)]$ is not bounded from below, then $u$ converges to 1 locally uniformly. And the reverse also holds. Then for bounded from below $E[u(\cdot, t)]$, the solution $u(x, t)$ converges to either 0 or $v(x)$. Finally, the convergence of $u(x, t)$ to 0 or $v(x)$ implies the corresponding convergence of energy.

Let us begin by assuming that $E[u(\cdot, t)]$ is not bounded from below. In this case, for cubic nonlinearity Flores proved in [12] that $\lim _{t \rightarrow \infty} u(x, t)=1$ locally uniformly by constructing a proper subsolution. Under (SD), we will 
prove a stronger conclusion. We will prove that if there exists $T \geq 0$ such that $E[u(\cdot, T)]<0$, then propagation occurs, in the sense defined in the introduction. Throughout the rest of this section, the assumptions of the above theorems are always assumed to be satisfied, and $u(x, t)$ always refers to the solutions of (1.1) and (1.2).

Lemma 3.2. Suppose there exists $c_{0}>0$ such that $\phi(x) \in H_{c_{0}}^{1}(\mathbb{R})$. If there exists $T \geq 0$ such that $E[u(\cdot, T)]<0$, then $u(x, t)$ is wave-like.

Proof. First observe that if $\phi(x) \in H_{c_{0}}^{1}(\mathbb{R})$, then $u(\cdot, T) \in H^{1}(\mathbb{R}) \cap H_{c_{0}}^{1}(\mathbb{R})$. Then for any small $\varepsilon>0$, if $E[u(x, T)]=-\varepsilon<0$ there exists $L>0$ such that $V(u(x, T)) \geq 0$ for $|x| \geq L$, and

$$
\begin{aligned}
& \int_{\{x \leq-L\}}\left(\frac{1}{2} u_{x}^{2}(x, T)+V(u(x, T))\right) d x<\frac{\varepsilon}{4}, \\
& \int_{\{x \geq L\}} e^{c_{0} x}\left(\frac{1}{2} u_{x}^{2}(x, T)+V(u(x, T))\right) d x<\frac{\varepsilon}{4} .
\end{aligned}
$$

Note that if we use smaller positive $c$ instead $c_{0}$ in the above inequality, the inequality still holds. And by the definition of $L$ we know that

$$
\int_{\{|x|<L\}}\left(\frac{1}{2} u_{x}^{2}(x, T)+V(u(x, T))\right) d x<-\varepsilon .
$$

So we can find a sufficiently small $c \in\left(0, c_{0}\right)$ such that

$$
\int_{\{|x|<L\}} e^{c x}\left(\frac{1}{2} u_{x}^{2}(x, T)+V(u(x, T))\right) d x<-\frac{\varepsilon}{2},
$$

and

$$
\Phi_{c}[u(\cdot, T)]=\int_{\mathbb{R}} e^{c x}\left(\frac{1}{2} u_{x}^{2}(x, T)+V(u(x, T))\right) d x<0 .
$$

So $u$ is wave-like.

We next show that for symmetric decreasing solutions and bistable nonlinearities the wave-like property also implies propagation in the sense of the introduction.

Lemma 3.3. Suppose that $u(x, t)$ is wave-like. Then $\lim _{t \rightarrow \infty} u(x, t)=1$ locally uniformly in $\mathbb{R}$.

Proof. In view of the definition of $\theta^{*}$ we have $V(u) \geq 0$ whenever $0 \leq u \leq \theta^{*}$, hence by $(2.9)$ we have $\delta_{0}>\theta^{*}$ in Proposition 2.7. Therefore, by that proposition

$$
R_{\theta^{*}}(t)>\frac{c t}{2}
$$

for sufficiently large $t$. Then, because $u(x, t)$ is symmetric decreasing, for any $L>0$ there exists $T_{L}>0$ such that $u(x, t)>\theta^{*}$ on the interval $[-L, L]$, for any $t \geq T_{L}$. Now, consider $\underline{u}(x, t)$ solving (1.1) with $\underline{u}\left(x, T_{L}\right)=\theta^{*}$ for all $x \in(-L, L)$ and $\underline{u}( \pm L, t)=\theta^{*}$ for all $t>T_{L}$. Since by our assumption on the nonlinearity the function $\underline{u}\left(x, T_{L}\right)$ is a strict subsolution, in the spirit of $[9$, 
Proposition 2.2] we have $\underline{u}(\cdot, t) \rightarrow v_{L}$ uniformly on $[-L, L]$, where $v_{L}$ solves (1.4) with $v_{L}( \pm L)=\theta^{*}$. Then, by comparison principle we obtain

$$
v_{L} \leq \liminf _{t \rightarrow \infty} u(\cdot, t) \leq \limsup _{t \rightarrow \infty} u(\cdot, t) \leq 1 \quad \text { uniformly in }[-L, L] .
$$

Also, by standard elliptic estimates we have $v_{L} \rightarrow \bar{v}$ locally uniformly as $L \rightarrow \infty$, where $\bar{v}$ solves (1.4) in the whole of $\mathbb{R}$. Since by construction $\bar{v} \geq \theta^{*}$, we have in fact $\bar{v}=1$. Then, passing to the limit in (3.12), we obtain the result.

Our next lemma uses a truncation argument to extend the conclusion of Lemma 3.3 to solutions that are not necessarily lying in any exponentially weighted Sobolev space, but have negative energy at some time $T \geq 0$.

Lemma 3.4. Suppose that there exists $T \geq 0$ such that $E[u(\cdot, T)]<0$, then $\lim _{t \rightarrow \infty} u(x, t)=1$ locally uniformly in $\mathbb{R}$.

Proof. For any $L>0$, we construct a cutoff function $\varphi_{L}(x)=\eta(|x| / L)$, where $\eta$ is a non-increasing $C^{\infty}(\mathbb{R})$ function such that $\eta(x)=1$ for $x<1$, and $\eta(x)=0$ for $x>2$. Let $\hat{\phi}(x ; L)=\varphi_{L}(x) u(x, T)$, so that $\hat{\phi}(x ; L) \rightarrow u(x, T)$ in $H^{1}(\mathbb{R})$ as $L \rightarrow \infty$. By our assumption and continuity of $E$, there exists a sufficiently large $L=L_{0}$, such that $E\left[\hat{\phi}\left(x ; L_{0}\right)\right]<0$. Note that $\hat{\phi}\left(x ; L_{0}\right)$ is a compactly supported function, so it lies in $H_{c}^{1}(\mathbb{R})$ for any $c>0$. Now consider the solution $\hat{u}(x, t)$ which satisfies (1.1) with initial condition $\hat{u}(x, 0)=\hat{\phi}\left(x ; L_{0}\right)$. From Lemma 3.3, we know that $\lim _{t \rightarrow \infty} \hat{u}(x, t)=1$ locally uniformly in $\mathbb{R}$. So by comparison principle $u(x, t+T) \geq \hat{u}(x, t)$, which proves the lemma.

An obvious corollary to the above lemma is the following.

Corollary 3.5. Suppose that $\lim _{t \rightarrow \infty} E[u(\cdot, t)]=-\infty$, then $\lim _{t \rightarrow \infty} u(x, t)=1$ locally uniformly in $\mathbb{R}$.

Our next lemma provides a sufficient condition for propagation, which, in particular, yields a conclusion converse to that of Corollary 3.5.

Lemma 3.6. Suppose that $\lim _{t \rightarrow \infty} u(x, t)=1$ locally uniformly in $\mathbb{R}$, then $\lim _{t \rightarrow \infty} E[u(\cdot, t)]=-\infty$.

Proof. We argue by contradiction. Suppose that $\lim _{t \rightarrow \infty} u(x, t)=1$ locally uniformly in $\mathbb{R}$ and $E[u(\cdot, t)]$ is bounded below. Then for any $L>0$, we can construct a cutoff function $\kappa_{L}(x)=\eta(|x|-L)$, where $\eta$ is defined in the proof of Lemma 3.4. For any $L>0, \kappa_{L}(x)=1$ for $|x|<L+1, \kappa(x)=0$ for $|x|>L+2$, and $\left|\kappa_{L}^{\prime}(x)\right|$ is bounded. Since $u(x, t)$ is symmetric decreasing, $\kappa_{L}$, $\kappa_{L}^{\prime}$ are both bounded, and $u, u_{x}$ are both bounded for all $t \geq 1$ by standard parabolic regularity, for $\tilde{u}_{L}(x, t):=\kappa_{L}(x) u(x, t)$ with any $t \geq 1$ we have the 
following energy estimate:

$$
\begin{aligned}
E\left[\tilde{u}_{L}(x, t)\right]= & 2 \int_{0}^{L+1} V(u) d x+\int_{0}^{L+1} u_{x}^{2} d x \\
& +\int_{L+1}^{L+2}\left\{\left(\frac{\partial\left(\kappa_{L} u\right)}{\partial x}\right)^{2}+2 V\left(\kappa_{L} u\right)\right\} d x \leq 2 \int_{0}^{L+1} V(u) d x+C,
\end{aligned}
$$

where the constant $C$ is independent of $L$. Since $\lim _{t \rightarrow \infty} u(x, t)=1$ locally uniformly in $\mathbb{R}$, for every $L_{0}>0$ satisfying $\left(L_{0}+1\right) V(1)<-C$, we can choose $t_{0}>0$ such that $V\left(u\left(x, t_{0}\right)\right)<V(1) / 2<0$ for any $x \in\left(-L_{0}-1, L_{0}+1\right)$. This implies that $\tilde{\phi}\left(x ; L_{0}\right)=\kappa_{L_{0}}(x) u\left(x, t_{0}\right)$ satisfies $E\left[\tilde{\phi}\left(x ; L_{0}\right)\right]<0$.

Note that $\tilde{\phi}\left(x ; L_{0}\right)$ is a compactly supported function, so it lies in $H_{c}^{1}(\mathbb{R})$ for any $c>0$. Now consider the solution $\tilde{u}(x, t)$ that satisfies $(1.1)$, with the initial condition $\tilde{u}(x, 0)=\tilde{\phi}\left(x ; L_{0}\right)$. By Proposition 2.7, Lemma 3.2, and the fact that

$$
u\left(x, t+t_{0}\right) \geq \tilde{u}(x, t), \quad x \in \mathbb{R}, t>0,
$$

there exists $c>0$ such that for any $t>t_{0}$,

$$
R_{\theta^{*}}>c\left(t-t_{0}\right)+R_{0}
$$

for some constant $R_{0} \in \mathbb{R}$. Moreover, we can find $T_{0}>0$ such that for any $t>T_{0}$ and $|x| \leq c t / 2$ we have

$$
u\left(x, t+t_{0}\right) \geq \theta^{*} .
$$

On the other hand, by (2.4) there exists a sufficiently large $t_{\alpha} \geq 0$ such that

$$
\int_{t_{\alpha}}^{\infty} \int_{\mathbb{R}} u_{t}^{2}(x, t) d x d t<\alpha^{2},
$$

for every $\alpha>0$. Let us take $\alpha=\theta_{0} \sqrt{c} / 9, t_{1}>\max \left\{t_{0}, t_{\alpha}\right\}$ and $x_{1}=R_{\theta_{0} / 2}\left(t_{1}\right)$. We also take $T>T_{0}$ such that $x_{1}<c T / 4$, and $t_{2}=t_{1}+T, x_{2}=x_{1}+c T$. Then by Cauchy-Schwarz inequality we have

$$
\begin{aligned}
\int_{t_{1}}^{t_{2}} \int_{x_{1}}^{x_{2}}\left|u_{t}(x, t)\right| d x d t & \leq \sqrt{\left(x_{2}-x_{1}\right)\left(t_{2}-t_{1}\right)}\left(\int_{t_{1}}^{t_{2}} \int_{x_{1}}^{x_{2}} u_{t}^{2}(x, t) d x d t\right)^{1 / 2} \\
& \leq \sqrt{c} T\left(\int_{t_{\alpha}}^{\infty} \int_{\mathbb{R}} u_{t}^{2}(x, t) d x d t\right)^{1 / 2} \\
& \leq \frac{c T \theta_{0}}{9}
\end{aligned}
$$

At the same time, since by construction $0<x_{1}<c T / 4$, we also have

$$
\begin{aligned}
\int_{t_{1}}^{t_{2}} \int_{x_{1}}^{x_{2}}\left|u_{t}(x, t)\right| d x d t & \geq \int_{c T / 4}^{c T / 2}\left(\int_{t_{1}}^{t_{2}}\left|u_{t}(x, t)\right| d t\right) d x \\
& \geq \int_{c T / 4}^{c T / 2}\left(u\left(x, t_{2}\right)-u\left(x, t_{1}\right)\right) d x
\end{aligned}
$$


Since $t_{2}>T>T_{0}$, we have $u\left(x, t_{2}\right) \geq \theta^{*}>\theta_{0}$ for $x \in(c T / 4, c T / 2)$. And by the definition of $x_{1}$ and $T$, we have $u\left(x, t_{1}\right)<\theta_{0} / 2$ for $x \in(c T / 4, c T / 2)$. So we have

$$
\int_{t_{1}}^{t_{2}} \int_{x_{1}}^{x_{2}}\left|u_{t}(x, t)\right| d x d t \geq \frac{c T \theta_{0}}{8},
$$

which contradicts (3.18).

Note that we have just proved the equivalence in part 1 of Theorem 2 . Indeed, we have a stronger corollary.

Corollary 3.7. We have $\lim _{t \rightarrow \infty} u(x, t)=1$ locally uniformly in $\mathbb{R}$, if and only if there exists $T \geq 0$ such that $E[u(\cdot, T)]<0$.

We now turn our attention to the case when $E[u(\cdot, t)]$ is bounded from below. By Lemmas 3.4 and 3.6, boundedness of $E[u(\cdot, t)]$ implies $\lim _{t \rightarrow \infty} E[u(\cdot, t)] \geq 0$. Below we prove that in this case either $\lim _{t \rightarrow \infty} u(x, t)=0$ uniformly in $\mathbb{R}$, or $\lim _{t \rightarrow \infty} u(x, t)=v(x)$ uniformly in $\mathbb{R}$. The idea of our proof is due to Fife [31, Lemma 10]. We refine Fife's arguments under our weaker assumptions on the nonlinearity and (SD).

The next lemma establishes existence of an increasing sequence $\left\{t_{n}\right\}$ tending to infinity on which the solution converges to a zero of $V(u)$ at the origin, thus allowing only two possibilities for the value of $\lim _{n \rightarrow \infty} u\left(0, t_{n}\right)$.

Lemma 3.8. If $E[u(\cdot, t)]$ is bounded from below, there exists an increasing sequence $\left\{t_{n}\right\}$ with $\lim _{n \rightarrow \infty} t_{n}=\infty$ such that either $\lim _{n \rightarrow \infty} u\left(0, t_{n}\right)=0$, or $\lim _{n \rightarrow \infty} u\left(0, t_{n}\right)=\theta^{*}$.

Proof. We multiply $u_{x}$ on both sides of Eq. (1.1), and integrate the products over $(-\infty, 0)$. Then we have

$$
\begin{aligned}
\int_{-\infty}^{0} u_{x}(x, t) u_{t}(x, t) d x & =\int_{-\infty}^{0}\left(u_{x x}(x, t)+f(u(x, t))\right) u_{x}(x, t) d x \\
& =\left.\frac{1}{2} u_{x}^{2}(x, t)\right|_{x=-\infty} ^{0}-(V(u(0, t))-V(u(-\infty, t))) \\
& =-V(u(0, t))
\end{aligned}
$$

From monotonicity of $u$ on $(-\infty, 0)$ and standard parabolic regularity, for $t \geq 1$ the left-hand side of (3.21) can be controlled by

$$
\begin{aligned}
\left|\int_{-\infty}^{0} u_{x}(x, t) u_{t}(x, t) d x\right| & \leq\left\|u_{t}(\cdot, t)\right\|_{L^{2}(-\infty, 0)}\left\|u_{x}(\cdot, t)\right\|_{L^{2}(-\infty, 0)} \\
& \leq\left\|u_{t}(\cdot, t)\right\|_{L^{2}(\mathbb{R})}\left\|u_{x}(\cdot, t)\right\|_{L^{\infty}(\mathbb{R})}^{1 / 2}|u(0, t)|^{1 / 2} \\
& \leq\left\|u_{t}(\cdot, t)\right\|_{L^{2}(\mathbb{R})}\left\|u_{x}\right\|_{L^{\infty}(\mathbb{R} \times(1, \infty))}^{1 / 2} \max \left\{1,\|\phi\|_{L^{\infty}(\mathbb{R})}^{1 / 2}\right\} .
\end{aligned}
$$


where we applied Cauchy-Schwarz inequality in the first line. Since $E[u(\cdot, t)]$ is bounded from below, by (2.4) we have

$$
\int_{1}^{\infty} \int_{\mathbb{R}} u_{t}^{2}(x, t) d x d t<\infty .
$$

Therefore, there exists an unbounded increasing sequence $\left\{t_{n}\right\}$ such that $\lim _{n \rightarrow \infty}\left\|u_{t}\left(\cdot, t_{n}\right)\right\|_{L^{2}(\mathbb{R})}=0$. Since also $\left\|u_{x}\right\|_{L^{\infty}(\mathbb{R} \times(1, \infty))}<\infty$ by standard parabolic regularity, this implies that $\lim _{n \rightarrow \infty} V\left(u\left(0, t_{n}\right)\right)=0$ by (3.21). Furthermore, since $\limsup \left\|u\left(\cdot, t_{n}\right)\right\|_{L^{\infty}(\mathbb{R})} \leq 1$, by the assumptions on the nonlinearity, $V(u)=0$ has only two roots, $u=0$ and $u=\theta^{*}$. Recall that for each $t>0$ the maximum of solution $u(\cdot, t)$ is always at the origin. By the structure of the nonlinearity $f$, we know that once $u(0, T)<\theta_{0}$ for some $T \geq 0$, then $\lim _{t \rightarrow \infty} u(x, t)=0$ uniformly in $\mathbb{R}$. Then, $\lim _{n \rightarrow \infty} V\left(u\left(0, t_{n}\right)\right)=0$ implies that either $\lim _{n \rightarrow \infty} u\left(0, t_{n}\right)=0$ or $\lim _{n \rightarrow \infty} u\left(0, t_{n}\right)=\theta^{*}$.

Lemma 3.9. The sequence $\left\{t_{n}\right\}$ in Lemma 3.8 can be chosen so as $t_{n+1}-t_{n} \leq 1$ for every $n$.

Proof. In view of (3.23), for each $n \in \mathbb{N}$ we have

$$
\lim _{n \rightarrow \infty} \int_{\frac{n}{2}}^{\frac{n+1}{2}} \int_{\mathbb{R}} u_{t}^{2}(x, t) d x d t=0 .
$$

Therefore, there exists $\left\{t_{n}\right\}$ with $t_{n} \in\left(\frac{n}{2}, \frac{n+1}{2}\right)$ such that $\left\|u_{t}\left(\cdot, t_{n}\right)\right\|_{L^{2}(\mathbb{R})} \rightarrow 0$ as $n \rightarrow \infty$, which yields the desired sequence.

In the proof of Lemma 3.8, we already obtained the uniform convergence corresponding to the first alternative.

Corollary 3.10. Suppose that there exists an increasing sequence $\left\{t_{n}\right\}$ such that $\lim _{n \rightarrow \infty} t_{n}=\infty$, and $\lim _{n \rightarrow \infty} u\left(0, t_{n}\right)=0$, then $\lim _{t \rightarrow \infty} u(x, t)=0$ uniformly in $\mathbb{R}$.

Combining the results of Lemmas 3.8 and 3.10, we now prove the following result.

Lemma 3.11. Suppose that $E[u(\cdot, t)]$ is bounded from below in $t$, then either $\lim _{t \rightarrow \infty} u(x, t)=0$, or $\lim _{t \rightarrow \infty} u(x, t)=v(x)$, uniformly in $\mathbb{R}$.

Proof. From Lemmas 3.8 and 3.10, we only need to prove that if the increasing sequence $\left\{t_{n}\right\}$ in Lemma 3.8 satisfies $\lim _{n \rightarrow \infty} u\left(0, t_{n}\right)=\theta^{*}$, then $\lim _{t \rightarrow \infty} u(x, t)=$ $v(x)$ uniformly in $\mathbb{R}$. To prove this, we first prove the locally uniform convergence on the sequence $\left\{t_{n}\right\}$. Let $w(x, t):=u(x, t)-v(x)$, then in view of $v(0)=\theta^{*}$ by Proposition 3.1 we have

$$
w_{t}=w_{x x}+f^{\prime}(\tilde{u}) w, \quad w_{x}(0, t)=0, \quad w(0, t)=u(0, t)-\theta^{*},
$$

where $\tilde{u}$ is between $u$ and $v$. We claim that

$$
\lim _{n \rightarrow \infty} w\left(x, t_{n}\right)=0,
$$


locally uniformly in $\mathbb{R}$. The proof follows from the continuous dependence on the data for solutions of the initial value problem in $x$ obtained from (3.25) for each $t=t_{n}$ fixed. Indeed, at $t=t_{n} \geq 1$ we denote $w_{n}(x):=w\left(x, t_{n}\right)$, $g_{n}(x):=u_{t}\left(x, t_{n}\right), K_{n}(x):=f^{\prime}\left(\tilde{u}\left(x, t_{n}\right)\right), \alpha_{n}:=u\left(0, t_{n}\right)-\theta^{*}$, and consider (3.25) as an ordinary differential equation in $x>0$ :

$$
w_{n}^{\prime \prime}=g_{n}-K_{n} w_{n}, \quad w_{n}^{\prime}(0)=0, \quad w_{n}(0)=\alpha_{n} .
$$

For any $L>0$, by integration over $(0, L)$ and an application of Cauchy-Schwarz inequality we have

$$
\begin{aligned}
\max _{0 \leq x \leq L}\left|w_{n}^{\prime}(x)\right| & \leq \sqrt{L}\left\|g_{n}\right\|_{L^{2}(\mathbb{R})}+L\left\|K_{n}\right\|_{L^{\infty}(\mathbb{R})} \max _{0 \leq x \leq L}\left|w_{n}(x)\right| \\
& \leq \sqrt{L}\left\|g_{n}\right\|_{L^{2}(\mathbb{R})}+L \mathcal{K} \max _{0 \leq x \leq L}\left|w_{n}(x)\right|,
\end{aligned}
$$

where the constant $\mathcal{K}$ satisfies

$$
\left|f^{\prime}(s)\right| \leq \mathcal{K}, \quad 0 \leq s \leq \max \left\{1,\|\phi(x)\|_{L^{\infty}(\mathbb{R})}\right\} .
$$

For fixed $L>0$, we choose a sufficiently large integer $l$ such that $2 \delta L \mathcal{K} \leq$ 1 for $\delta:=L / l$. We next take

$$
\begin{aligned}
& W_{n, k}:=\max _{(k-1) \delta \leq x \leq k \delta}\left|w_{n}(x)\right|, \quad k \in \mathbb{N}, \\
& m_{n, 0}:=\alpha_{n}, \quad m_{n, k}:=\max _{1 \leq k^{\prime} \leq k} W_{n, k^{\prime}} .
\end{aligned}
$$

Then $m_{n, k}$ is non-decreasing in $k$, and $m_{n, k}=\max _{0 \leq x \leq k \delta}\left|w_{n}(x)\right|$. By (3.28) and our choice of $\delta$, for any $1 \leq k \leq l$ we have

$$
\begin{aligned}
m_{n, k}-m_{n, k-1} & \leq \delta \max _{0 \leq x \leq L}\left|w_{n}^{\prime}(x)\right| \\
& \leq \delta\left(\sqrt{L}\left\|g_{n}\right\|_{L^{2}(\mathbb{R})}+L \mathcal{K} m_{n, k}\right) \\
& \leq \delta \sqrt{L}\left\|g_{n}\right\|_{L^{2}(\mathbb{R})}+\frac{m_{n, k}}{2} .
\end{aligned}
$$

This implies that for any $1 \leq k \leq l$ we have

$$
m_{n, k} \leq 2 m_{n, k-1}+G_{n},
$$

where $G_{n}:=2 \delta \sqrt{L}\left\|g_{n}\right\|_{L^{2}(\mathbb{R})}$. Since by definition $m_{n, 0}=\alpha_{n}$, by iteration and symmetry of $w_{n}(x)$ we have

$$
\max _{-L \leq x \leq L}\left|w_{n}(x)\right|=m_{n, l} \leq 2^{l} \alpha_{n}+\left(2^{l}-1\right) G_{n} .
$$

Now, as $n \rightarrow \infty$, by Lemmas 3.8 and 3.9 we know that $u\left(0, t_{n}\right)-\theta^{*} \rightarrow 0$ and $\left\|u_{t}\left(x, t_{n}\right)\right\|_{L^{2}(\mathbb{R})} \rightarrow 0$, so that $\alpha_{n} \rightarrow 0, G_{n} \rightarrow 0$, and $\max _{-L \leq x \leq L}\left|w_{n}(x)\right| \rightarrow 0$, i.e., $u\left(x, t_{n}\right)$ converges to $v(x)$ locally uniformly. Then by Proposition 2.8 and the fact that by Lemma 3.9 the sequence $\left\{t_{n}\right\}$ can be chosen so as $t_{n+1}-t_{n} \leq 1$, we can obtain the full limit convergence. Indeed, since the Hölder constant in $t$ of $u(x, t)$ converges to 0 as $n \rightarrow \infty$ uniformly for all $|x| \leq L$ and all $t_{n}<t<t_{n+1}$, we have

$$
|u(x, t)-v(x)| \leq\left|u\left(x, t_{n}\right)-v(x)\right|+\left|u(x, t)-u\left(x, t_{n}\right)\right| \rightarrow 0 \quad \text { as } n \rightarrow \infty .
$$


Finally, let us prove that convergence of $u(x, t)$ to $v(x)$ is, in fact, uniform. Indeed, since $u(x, t)$ is symmetric decreasing in $x$ and $v(x) \rightarrow 0$ as $|x| \rightarrow \infty$, for any $L>0, t>0$ we have

$$
\begin{aligned}
\sup _{|x| \geq L}|w(x, t)| & =\sup _{|x| \geq L}|u(x, t)-v(x)| \\
& \leq \max _{|x| \geq L}\{u(x, t), v(x)\} \\
& \leq \max \{u(L, t), v(L)\} \\
& \leq v(L)+\max _{|x| \leq L}|w(x, t)| .
\end{aligned}
$$

This implies that

$$
\sup _{x \in \mathbb{R}}|w(x, t)| \leq v(L)+\max _{|x| \leq L}|w(x, t)| .
$$

Then, for any $\varepsilon>0$ we can find $L>0$ sufficiently large such that $v(L)<\varepsilon / 2$. We can also find $T>0$ such that $|w(x, t)|<\varepsilon / 2$ for any $x \in[-L, L], t>T$. So we get

$$
\lim _{t \rightarrow \infty}|w(x, t)|=0,
$$

uniformly in $x \in \mathbb{R}$, which proves the lemma.

Note that in view of the results in the preceding lemmas, by proving Lemma 3.11 we have just proved Theorem 1.

Remark 3.12. By standard parabolic regularity, under the assumptions of Lemma 3.11 we also have

$$
\lim _{t \rightarrow \infty} u(x, t)=v(x) \quad \text { in } C^{1}(\mathbb{R}) .
$$

We now turn to the study of the limit value of energy. At first, we prove that the energy of the solution goes to zero, if extinction occurs.

Lemma 3.13. If $\lim _{t \rightarrow \infty} u(x, t)=0$ uniformly in $\mathbb{R}$, then $\lim _{t \rightarrow \infty} E[u(\cdot, t)]=0$.

Proof. From condition (SD), we have

$$
\begin{aligned}
\int_{\mathbb{R}} \frac{1}{2} u_{x}^{2}(x, t) d x & =\int_{0}^{\infty} u_{x}^{2}(x, t) d x \\
& \leq\left\|u_{x}(x, t)\right\|_{L^{\infty}(\mathbb{R})} u(0, t) .
\end{aligned}
$$

By standard parabolic regularity, if $\lim _{t \rightarrow \infty} u(x, t)=0$ uniformly in $\mathbb{R}$, then

$$
\lim _{t \rightarrow \infty} \int_{\mathbb{R}} \frac{1}{2} u_{x}^{2}(x, t) d x \rightarrow 0 .
$$

So we only need to show that $\lim _{t \rightarrow \infty} \int_{\mathbb{R}} V(u(x, t)) d x=0$.

If $f^{\prime}(0)<0$, there exists $C>0$ such that

$$
0 \leq V(u) \leq C u^{2}
$$


for small enough $u$. Then from the usual energy estimate we obtain $\lim _{t \rightarrow \infty}\|u(\cdot, t)\|_{L^{2}(\mathbb{R})}^{2}=0$ exponentially, so that $\lim _{t \rightarrow \infty} \int_{\mathbb{R}} V(u(x, t)) d x=0$ as well. Alternatively, if $f^{\prime}(0)=0$, then by (3.4) we have

$$
0 \leq V(u) \leq C u^{p+1}
$$

for some $C>0$ and sufficiently small $u$. So it is enough to show that $\lim _{t \rightarrow \infty}\|u(\cdot, t)\|_{L^{p+1}(\mathbb{R})}^{p+1}=0$. In view of $(3.4)$ we can use the solution $\bar{u}(x, t)$ of the heat equation:

$$
\bar{u}_{t}=\bar{u}_{x x}, \quad x \in \mathbb{R}, \quad t>T, \quad \bar{u}(x, T)=u(x, T), \quad x \in \mathbb{R},
$$

as a supersolution to obtain (see, e.g., [21, Proposition 48.4])

$$
\begin{aligned}
& \|u(\cdot, t)\|_{L^{p+1}(\mathbb{R})} \leq\|\bar{u}(\cdot, t)\|_{L^{p+1}(\mathbb{R})} \leq\left((4 \pi(t-T))^{-\frac{p-1}{4(p+1)}}\|u(\cdot, T)\|_{L^{2}(\mathbb{R})} \rightarrow 0\right. \\
& \quad \text { as } t \rightarrow \infty,
\end{aligned}
$$

and the statement follows.

If, on the other hand, $\lim _{t \rightarrow \infty} u(x, t)=v(x)$ uniformly in $\mathbb{R}$, then we claim that $E[u(\cdot, t)]$ has a limit as $t \rightarrow \infty$, and the value of the limit is equal to $E_{0}$ defined in Proposition 3.1. We begin with the analysis of the non-degenerate case.

Lemma 3.14. Suppose that $f^{\prime}(0)<0$, then $\lim _{t \rightarrow \infty} u(x, t)=v(x)$ uniformly in $\mathbb{R}$ implies $\lim _{t \rightarrow \infty} E[u(\cdot, t)]=E_{0}$.

Proof. At first, we show that for any fixed $L>0$, the energy $E[u(\cdot, t) ; L]$ of $u(x, t)$ restricted to $[-L, L]$, namely $E[u(\cdot, t) ; L]:=\int_{-L}^{L}\left(\frac{1}{2} u_{x}^{2}+V(u)\right) d x$, converges to the energy $E[v ; L]$ of $v(x)$ restricted to $[-L, L]$. Then we show that $E[u(\cdot, t)]-E[u(\cdot, t) ; L]$ converges to $E_{0}-E[v ; L]$ for sufficiently large $L$.

Since upon integration of (1.4) we have $\left|v^{\prime}\right|=\sqrt{2 V(u)}$, on the interval $[-L, L]$ we can compute

$$
E[v ; L]=\int_{-L}^{L}\left(\frac{1}{2}\left|v^{\prime}\right|^{2}+V(v)\right) d x=2 \sqrt{2} \int_{v(L)}^{\theta^{*}} \sqrt{V(u)} d u .
$$

We also know that $u(x, t) \rightarrow v(x), u_{x}(x, t) \rightarrow v^{\prime}(x)$ uniformly in $x \in$ $[-L, L]$, as $t \rightarrow \infty$, by Lemma 3.12. This implies that

$$
\lim _{t \rightarrow \infty} E[u(\cdot, t) ; L]=E[v ; L] .
$$

By symmetry of the solution, the remaining part of energy can be estimated as follows:

$$
E[u(\cdot, t)]-E[u(\cdot, t) ; L]=\int_{L}^{\infty}\left(u_{x}^{2}(x, t)+2 V(u(x, t))\right) d x .
$$

And by decrease of the solution for $x>0$ we know that

$$
\int_{L}^{\infty} u_{x}^{2}(x, t) d x \leq u(L, t)\left\|u_{x}(x, t)\right\|_{L^{\infty}(\mathbb{R})}
$$


By standard parabolic regularity, for $t \geq 1$, the above expression converges to 0 as $L \rightarrow \infty$. In addition, we have $E[v]-E[v ; L] \rightarrow 0$ as $L \rightarrow \infty$. So we only need to show that for any $\delta>0$ there exist a sufficiently large $L_{\delta}>0$, $T_{\delta}>0$ such that for any $t>T_{\delta}$,

$$
\left|\int_{L_{\delta}}^{\infty} V(u(x, t)) d x\right|<\delta .
$$

If $f^{\prime}(0)<0$, then there exists $K>0$ such that $f(u) \leq-K u$ for all $u \in\left[0, \theta_{0} / 2\right]$. We can then finish the proof of the lemma by an $L^{2}$ decay estimate similar to the one in the proof of Lemma 3.13. Taking $L>0$ satisfying $v(L)<\theta_{0} / 4$, there exists $T>0$ such that $u(x, t)<\theta_{0} / 2$ for any $x \in(L, \infty)$ and any $t>T$. Then for $t>T$ we have

$$
\begin{aligned}
\frac{d}{d t} \int_{L}^{\infty} u^{2} d x & =2 \int_{L}^{\infty} u(x, t)\left(u_{x x}(x, t)+f(u)\right) d x \\
& \leq 2 u(L, t)\left|u_{x}(L, t)\right|-2 K \int_{L}^{\infty} u^{2}(x, t) d x .
\end{aligned}
$$

Since $\lim _{t \rightarrow \infty} u(L, t)\left|u_{x}(L, t)\right|=v(L)\left|v^{\prime}(L)\right|$, from the above inequality and the relation $0 \leq V(u(x, t)) \leq C u^{2}$ on $u \in\left[0, \theta_{0}\right]$ for some $C>0$, we know that there exists $\hat{T}>T$ such that for any $t>\hat{T}$

$$
0 \leq \int_{L}^{\infty} V(u(x, t)) d x<\frac{2 C v(L)\left|v^{\prime}(L)\right|}{K} .
$$

Since $v(L) v^{\prime}(L) \rightarrow 0$ as $L \rightarrow \infty$, we have the desired conclusion.

Now to the degenerate case.

Lemma 3.15. If $f^{\prime}(0)=0$, then $\lim _{t \rightarrow \infty} u(x, t)=v(x)$ uniformly in $\mathbb{R}$ implies $\lim _{t \rightarrow \infty} E[u(\cdot, t)]=E_{0}$, when (3.3) and (3.4) hold.

Proof. In the spirit of Lemma 3.14, we only need to show that

$$
\limsup _{t \rightarrow \infty} \int_{L}^{\infty} V(u(x, t)) d x \rightarrow 0 \quad \text { as } L \rightarrow \infty .
$$

By (3.4), for any sufficiently small $\delta>0$ we have

$$
0 \leq V(u) \leq \frac{2 k u^{p+1}}{p+1} \quad \forall u \in[0, \delta]
$$

Furthermore, by Proposition 3.1 we can fix $L \sim \delta^{-\frac{p-1}{2}} \gg 1$ such that $v(L)=$ $\delta / 2$. Because $u(L, t)$ converges to $v(L)$ as $t \rightarrow \infty$, for sufficiently large $t$ we have $u(x, t) \leq \delta$ for all $x \geq L$ and

$$
0 \leq \int_{L}^{\infty} V(u(x, t)) d x \leq \frac{2 k}{p+1}\|u(\cdot, t)\|_{L^{p+1}(L, \infty)}^{p+1} .
$$

Then we only need to control $\|u(\cdot, t)\|_{L^{p+1}(L, \infty)}$ by $\delta$ for large enough $t$. 
We denote by $\bar{v}(x)$ a shift of the bump solution $v(x)$ from Proposition 3.1 which satisfies $0<\bar{v} \leq \delta$ for all $x>L$ and

$$
\left\{\begin{array}{l}
0=\bar{v}^{\prime \prime}+f(\bar{v}), \quad x>L \\
\bar{v}(L)=\delta \\
\bar{v}(\infty)=0
\end{array}\right.
$$

Then we construct a supersolution $\bar{u}$, which solves the half-line problem:

$$
\left\{\begin{array}{l}
\bar{u}_{t}=\bar{u}_{x x}+f(\bar{u}), \quad x>L, t>T, \\
\bar{u}(L, t)=\delta \\
\bar{u}(x, T)=\max \{u(x, T), \bar{v}(x)\} .
\end{array}\right.
$$

Note that since $\hat{u}(x, t) \equiv \delta$ is a supersolution for $\bar{u}(x, t)$, we have $\bar{u}(x, t) \leq \delta$ for all $x \geq L$ and $t \geq T$. And by comparison principle we have $u(x, t) \leq \bar{u}(x, t)$ for all $x \geq L$ and $t \geq T$.

We now introduce

$$
w(x, t):=\bar{u}(x, t)-\bar{v}(x) \geq 0, \quad x>L, \quad t>T,
$$

which satisfies the linear equation:

$$
w_{t}=w_{x x}+f^{\prime}(\tilde{w}) w, \quad x>L, \quad t>T,
$$

for some $\bar{v} \leq \tilde{w} \leq \bar{u}$, with homogeneous Dirichlet boundary condition

$$
w(L, t)=0, \quad t>T .
$$

Since $0 \leq w(x, T) \leq u(x, T)$, we have $w(\cdot, T) \in L^{2}(\mathbb{R})$ by Proposition 2.1. Furthermore, in view of (3.3) the solution $\bar{w}$ of the heat equation with the same initial and boundary conditions:

$\bar{w}_{t}=\bar{w}_{x x}, \quad x>L, \quad t>T, \quad \bar{w}(L, t)=0, \quad t>T, \quad \bar{w}(x, T)=w(x, T), x>L$,

is a supersolution for $w$. Then, by the estimate similar to the one in (3.45) and comparison principle, we have:

$$
\begin{aligned}
& \|w(\cdot, t)\|_{L^{p+1}(\mathbb{R})} \leq\|\bar{w}(\cdot, t)\|_{L^{p+1}(\mathbb{R})} \leq C(t-T)^{-\frac{p-1}{4(p+1)}}\|w(\cdot, T)\|_{L^{2}(\mathbb{R})} \rightarrow 0 \\
& \quad \text { as } t \rightarrow \infty
\end{aligned}
$$

Estimating $\|\bar{u}(\cdot, t)\|_{L^{p+1}(\mathbb{R})}$ in terms of $\|w(\cdot, t)\|_{L^{p+1}(\mathbb{R})}$, we obtain

$$
\|\bar{u}(\cdot, t)\|_{L^{p+1}(L, \infty)} \leq\|w(\cdot, t)\|_{L^{p+1}(L, \infty)}+\|\bar{v}\|_{L^{p+1}(L, \infty)} \quad \forall t \geq T .
$$

On the other hand, it is clear that the estimates in Proposition 3.1 apply to $\bar{v}$ as well. Therefore

$$
\|\bar{v}\|_{L^{p+1}(L, \infty)}^{p+1} \leq C \delta^{\frac{p+3}{2}} .
$$

for some $C>0$ and all $\delta>0$ sufficiently small. Finally, combining (3.62) and (3.64) in (3.63), by comparison principle we conclude that $\|u(\cdot, t)\|_{L^{p+1}(L, \infty)}$ can be made arbitrarily small for all $t \geq T$ by choosing a sufficiently small $\delta$ in the limit $t \rightarrow \infty$. 
Note that we have now proved our Theorem 2.

Let us finally consider the question of threshold phenomena. We use similar notations as in [13]. Let $X:=\{\phi(x): \phi(x)$ satisfies (1.2) and (SD) $\}$. We consider a one-parameter family of initial conditions $\phi_{\lambda}, \lambda>0$, satisfying the following conditions:

(P1) For any $\lambda>0, \phi_{\lambda} \in X$, the map $\lambda \mapsto \phi_{\lambda}$ is continuous from $\mathbb{R}_{+}$to $L^{2}(\mathbb{R})$;

(P2) If $0<\lambda_{1}<\lambda_{2}$, then $\phi_{\lambda_{1}} \leq \phi_{\lambda_{2}}$ and $\phi_{\lambda_{1}} \neq \phi_{\lambda_{2}}$ in $L^{2}(\mathbb{R})$.

(P3) $\lim _{\lambda \rightarrow 0} \phi_{\lambda}(x)=0$ in $L^{2}(\mathbb{R})$.

We denote by $u_{\lambda}(x, t)$ the solution of (1.1) with the initial datum $\phi_{\lambda}$.

Here is our main result concerning threshold phenomena for bistable nonlinearities.

Theorem 3. Under the same conditions as in Theorem 1, suppose that (P1) through (P3) hold. Then one of the following two conclusions is true:

1. $\lim _{t \rightarrow \infty} u_{\lambda}(x, t)=0$ uniformly in $\mathbb{R}$ for every $\lambda>0$;

2. There exists $\lambda^{*}>0$ such that

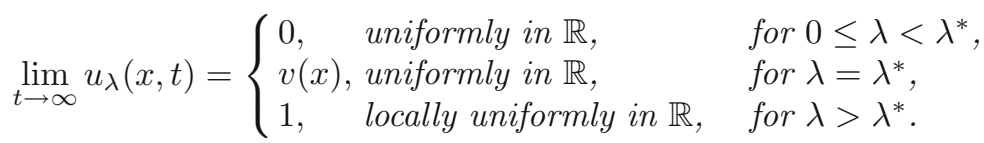

Proof. We define

$$
\Sigma_{0}:=\left\{\lambda>0: u_{\lambda}(x, t) \rightarrow 0 \text { as } t \rightarrow \infty \text { uniformly in } x \in \mathbb{R}\right\},
$$

$$
\Sigma_{1}:=\left\{\lambda>0: u_{\lambda}(x, t) \rightarrow 1 \text { as } t \rightarrow \infty \text { locally uniformly in } x \in \mathbb{R}\right\} .
$$

We know that $\lambda \in \Sigma_{0}$ if and only if there exists $T \geq 0$ such that $u(0, T)<\theta_{0}$. Clearly the set $\Sigma_{0}$ is open. Furthermore, by comparison principle, if $\hat{\lambda} \in \Sigma_{0}$, then for any $\lambda<\hat{\lambda}, \lambda \in \Sigma_{0}$. So $\Sigma_{0}$ is an open interval.

If $\Sigma_{1} \neq(0, \varnothing)$, then the set $\Sigma_{1}$ is an open interval (semi-infinite) as well. Indeed, by Corollary 3.7 for every $\lambda \in \Sigma_{1}$ there exists $T \geq 0$ such that $E\left[u_{\lambda}(\cdot, T)\right]<0$. Then by continuity of the energy functional in $H^{1}(\mathbb{R})$ and continuous dependence in $H^{1}(\mathbb{R})$ of the solution at $t>0$ on the initial data in $L^{2}(\mathbb{R})$ (see Proposition 2.1), there exists $\delta>0$ such that for all $\left|\lambda^{\prime}-\lambda\right|<\delta$ we have $E\left[u_{\lambda^{\prime}}(\cdot, T)\right]<0$. Hence $\lambda^{\prime} \in \Sigma_{1}$ as well. And by comparison principle, if $\tilde{\lambda} \in \Sigma_{1}$, then for any $\lambda>\tilde{\lambda}, \lambda \in \Sigma_{1}$. Then we know that $\mathbb{R}_{+} \backslash\left(\Sigma_{0} \cup \Sigma_{1}\right)$ is a closed set, and, more precisely, a closed interval.

We will prove that if $\mathbb{R}_{+} \backslash\left(\Sigma_{0} \cup \Sigma_{1}\right)$ is not empty, then it contains only one point. Consider the Schrödinger-type operator:

$$
\mathfrak{L}=-\frac{d^{2}}{d x^{2}}+\mathcal{V}(x), \quad \mathcal{V}(x):=-f^{\prime}(v(x)),
$$

and the associated Rayleigh quotient (for technical background, see, e.g., [32, Chapter 11]):

$$
\mathfrak{R}(\phi):=\frac{\int_{\mathbb{R}}\left(\left|\phi^{\prime}\right|^{2}+\mathcal{V}(x) \phi^{2}\right) d x}{\int_{\mathbb{R}} \phi^{2} d x} .
$$


To study minimization problem for $\mathfrak{R}$, we also consider

$$
\tilde{\mathfrak{L}}=-\frac{d^{2}}{d x^{2}}+\tilde{\mathcal{V}}(x), \quad \tilde{\mathcal{V}}(x):=\mathcal{V}(x)+f^{\prime}(0)=-\left(f^{\prime}(v(x))-f^{\prime}(0)\right),
$$

with the associated Rayleigh quotient

$$
\tilde{\mathfrak{R}}(\phi)=\mathfrak{R}(\phi)+f^{\prime}(0) .
$$

Since $\tilde{\mathcal{V}}(x) \in L^{\infty}(\mathbb{R})$ and vanishes at infinity, by [32, Theorem 11.5] there exists a function $\phi_{0} \in H^{1}(\mathbb{R})$ such that $\phi_{0} \neq 0$ and $\phi_{0}$ minimizes $\tilde{\mathfrak{R}}$, provided

$$
\mathfrak{E}_{0}:=\inf \left\{\tilde{\mathfrak{R}}(\phi): \phi \in H^{1}(\mathbb{R}), \phi \neq 0\right\}<0 .
$$

Moreover, by [32, Theorem 11.8], if there exists a minimizer $\phi_{0} \in H^{1}(\mathbb{R})$, $\phi_{0} \neq 0$, then $\phi_{0}$ can be chosen to be a strictly positive function, and $\phi_{0}$ is unique up to a constant factor. Then, since by Proposition 3.1 we have $v^{\prime} \in H^{1}(\mathbb{R})$, translational symmetry of the problem yields (test (1.4) with $v^{\prime \prime}$ and integrate by parts):

$$
\tilde{\mathfrak{R}}\left(v^{\prime}\right)=f^{\prime}(0) .
$$

By $(3.3), f^{\prime}(0) \leq 0$. Moreover, since $v^{\prime}$ changes sign, we know that $v^{\prime}$ is not a minimizer of $\tilde{\mathfrak{R}}$, so $\mathfrak{E}_{0}<\tilde{\mathfrak{R}}\left(v^{\prime}\right) \leq 0$, and there exists a positive function $\phi_{0} \in H^{1}(\mathbb{R})$ that minimizes $\tilde{\Re}$, with

$$
\min \left\{\tilde{\mathfrak{R}}(\phi): \phi \in H^{1}(\mathbb{R}), \phi \neq 0\right\}=\tilde{\mathfrak{R}}\left(\phi_{0}\right)<f^{\prime}(0) .
$$

Note that $\phi_{0}$ also minimizes $\mathfrak{R}$, with

$$
\min \left\{\mathfrak{R}(\phi): \phi \in H^{1}(\mathbb{R}), \phi \neq 0\right\}=\mathfrak{R}\left(\phi_{0}\right)=: \nu_{0}<0 .
$$

Approximating $\phi_{0}$ by a function with compact support and using it as a test function, we can then see that

$$
\min \left\{\mathfrak{R}(\phi): \phi \in H_{0}^{1}(-L, L), \phi \neq 0\right\}=: \nu_{0}^{L}<0
$$

as well for a sufficiently large $L>0$, and in this case there exists a positive minimizer $\phi_{0}^{L} \in H_{0}^{1}(-L, L) \cap C^{2}(-L, L) \cap C^{1}([-L, L])$ such that

$$
\mathfrak{L}\left(\phi_{0}^{L}\right)=\nu_{0}^{L} \phi_{0}^{L} .
$$

If $\Sigma_{1}$ is not empty and the threshold set $\mathbb{R}_{+} \backslash\left(\Sigma_{0} \cup \Sigma_{1}\right)$ does not contain only one point, then there exist two distinct values $0<\lambda_{1}<\lambda_{2}$ in the threshold set. Since $f(u) \in C^{1}([0, \infty)), f^{\prime}(u)$ is uniformly continuous on $\left[0, \max \left\{1,\|\phi\|_{L^{\infty}}\right\}\right]$. Thus, there exists $\delta>0$ such that

$$
\left|f^{\prime}\left(u_{1}\right)-f^{\prime}\left(u_{2}\right)\right|<\frac{\left|\nu_{0}^{L}\right|}{2}
$$

for any $u_{1}, u_{2} \in\left[0, \max \left\{1,\|\phi\|_{L^{\infty}}\right\}\right]$ satisfying $\left|u_{1}-u_{2}\right|<\delta$. Since $\lambda_{1,2} \in$ $\mathbb{R}_{+} \backslash\left(\Sigma_{0} \cup \Sigma_{1}\right)$, we have $\lim _{t \rightarrow \infty} u_{\lambda_{1,2}}(x, t)=v(x)$ uniformly in $x \in \mathbb{R}$. Then, there exists $T$ sufficiently large, such that $\left|u_{\lambda_{1,2}}(x, t)-v(x)\right|<\delta$ for any $t \geq T$ and all $x \in \mathbb{R}$. So we have

$$
\max _{x \in[-L, L]}\left|f^{\prime}(v(x))-f^{\prime}(\tilde{u}(x, t))\right|<\frac{\left|\nu_{0}^{L}\right|}{2},
$$


for every $u_{\lambda_{1}}(x, t) \leq \tilde{u}(x, t) \leq u_{\lambda_{2}}(x, t)$ and all $t \geq T$. However, let $w(x, t)=$ $u_{\lambda_{2}}(x, t)-u_{\lambda_{1}}(x, t)$, then $w(x, t)$ satisfies the following equation,

$$
w_{t}=w_{x x}+f^{\prime}(\tilde{u}) w, \quad x \in \mathbb{R}, \quad t>0,
$$

for some $u_{\lambda_{1}}(x, t) \leq \tilde{u}(x, t) \leq u_{\lambda_{2}}(x, t)$. By the strong maximum principle $w(x, t)>0$ for any $x \in \mathbb{R}$ and $t>0$. Hence there exists $\varepsilon>0$ such that $w(x, T)>\varepsilon \phi_{0}^{L}(x)$. Let $\varepsilon \phi_{0}^{L}(x)=: \underline{w}(x, t)$. Then

$$
\begin{aligned}
\underline{w}_{t}-\underline{w}_{x x}-f^{\prime}(\tilde{u}) \underline{w} & =-\underline{w}_{x x}-f^{\prime}(v) \underline{w}+\left(f^{\prime}(v)-f^{\prime}(\tilde{u})\right) \underline{w} \\
& =\nu_{0}^{L} \underline{w}+\left(f^{\prime}(v)-f^{\prime}(\tilde{u})\right) \underline{w} \\
& \leq \frac{\nu_{0}^{L}}{2} \underline{w} \\
& \leq 0,
\end{aligned}
$$

which implies that $\underline{w}(x, t)$ is a subsolution for $t \geq T$. So by comparison principle

$$
u_{\lambda_{2}}-u_{\lambda_{1}} \geq \varepsilon \phi_{0}^{L}(x), \quad \forall t \geq T,
$$

i.e., there exists a barrier between $u_{\lambda_{1}}$ and $u_{\lambda_{2}}$, which contradicts the assumption that both $u_{\lambda_{1}}(x, t)$ and $u_{\lambda_{2}}(x, t)$ converge to $v(x)$ uniformly in $\mathbb{R}$, as $t \rightarrow \infty$. It means that if $\mathbb{R}_{+} \backslash\left(\Sigma_{0} \cup \Sigma_{1}\right)$ is not empty, then it only contains one point.

Remark 3.16. By Corollary 3.7 and comparison principle, to ensure that $\lambda^{*}<$ $\infty$ in Theorem 3 it is enough if there exists $\lambda>0$ and $\tilde{\phi}_{\lambda} \in L^{2}(\mathbb{R})$ such that $0 \leq \tilde{\phi}_{\lambda} \leq \phi_{\lambda}$ and $E\left[\tilde{\phi}_{\lambda}\right]<0$. This condition is easily seen to be verified for the family of characteristic functions of growing symmetric intervals studied by Kanel' [8]. Also, by Theorem 2 and the monotone decrease of the energy evaluated on solutions the condition $E\left[\phi_{\lambda}\right]<E_{0}$ for some $\lambda>0$ implies that $u_{\lambda}(x, t) \nrightarrow v(x)$. In particular, if $\sup _{0<\lambda<\bar{\lambda}} E\left[\phi_{\lambda}\right]<E_{0}$, then $\lambda^{*}>\bar{\lambda}$.

\section{Monostable Nonlinearity}

In this section, we study the monostable nonlinearity, i.e., $f(u) \in C^{1}([0, \infty), \mathbb{R})$,

$$
f(0)=f(1)=0, \quad f(u) \begin{cases}>0, & \text { in }(0,1), \\ <0, & \text { in }(1, \infty) .\end{cases}
$$

Moreover, we assume that the monostable nonlinearity $f(u)$ also satisfies

$$
f^{\prime}(0)=0 \text {. }
$$

Typical examples are the Arrhenius combustion nonlinearity

$$
f(u)=(1-u) e^{-\frac{a}{u}}, \quad a>0,
$$

and the generalized Fisher nonlinearity, i.e., the nonlinearity

$$
f(u)=u^{p}(1-u),
$$

with exponent $p>1$. 
Under conditions (4.1), there exists one root of $V(u): u=0$, and possibly a second root $u=\theta^{\diamond}>1$. However, since $\lim _{t \rightarrow \infty}\|u(x, t)\|_{L^{\infty}(\mathbb{R})} \leq 1$, without loss of generality, we suppose that $\|\phi\|_{L^{\infty}(\mathbb{R})}<\theta^{\diamond}$. So that we always suppose that $V(u) \leq 0$.

We have the following theorems about convergence and one-to-one relations between the limit value of the energy and the long time behavior of solutions, similar to the bistable case.

Theorem 4. Let $f$ satisfy conditions (4.1) and (4.2), and let $\phi(x)$ satisfy condition (1.3) and hypothesis (SD). Then one of the following holds.

1. $\lim _{t \rightarrow \infty} u(x, t)=1$ locally uniformly in $\mathbb{R}$,

2. $\lim _{t \rightarrow \infty} u(x, t)=0$ uniformly in $\mathbb{R}$.

Theorem 5. Under the same conditions as in Theorem 4, we have the following one-to-one relation.

1. $\lim _{t \rightarrow \infty} u(x, t)=1$ locally uniformly in $\mathbb{R} \Leftrightarrow \lim _{t \rightarrow \infty} E[u(\cdot, t)]=-\infty$.

2. $\lim _{t \rightarrow \infty} u(x, t)=0$ uniformly in $\mathbb{R} \Leftrightarrow \lim _{t \rightarrow \infty} E[u(\cdot, t)]=0$.

Throughout the rest of this section, the hypotheses of Theorem 4 are assumed to be satisfied. We start by establishing the following conclusion.

Lemma 4.1. If $\lim _{t \rightarrow \infty} u(x, t)=1$ locally uniformly in $\mathbb{R}$, then $\lim _{t \rightarrow \infty} E[u(\cdot, t)]=$ $-\infty$. And if $\lim _{t \rightarrow \infty} u(x, t)=0$ uniformly in $\mathbb{R}$, then $\lim _{t \rightarrow \infty} E[u(\cdot, t)] \leq 0$.

Proof. Under condition (4.1), we know that $\int_{\mathbb{R}} V(u) d x \leq 0$. And if $u \rightarrow 1$ locally uniformly in $\mathbb{R}$, then

$$
\lim _{t \rightarrow \infty} \int_{\mathbb{R}} V(u(x, t)) d x=-\infty .
$$

By hypothesis (SD), we have

$$
\begin{aligned}
\int_{\mathbb{R}} \frac{1}{2} u_{x}^{2}(x, t) d x & =\int_{0}^{\infty} u_{x}^{2}(x, t) d x \\
& \leq\left\|u_{x}(x, t)\right\|_{L^{\infty}(\mathbb{R})} u(0, t) .
\end{aligned}
$$

Then by standard parabolic regularity the left-hand side of (4.6) is bounded uniformly in time. So we proved the first conclusion. On the other hand, if $u \rightarrow 0$ uniformly in $\mathbb{R}$, then the left-hand side of (4.6) converges to 0 . In view of $V(u(x, t)) \leq 0$, we proved the second conclusion.

Similarly to Lemma 3.2 for the bistable case, we have the following lemma for the monostable case.

Lemma 4.2. Assume that there exists $c_{0}>0$ such that $\phi(x) \in H_{c_{0}}^{1}(\mathbb{R})$. If there exists $T \geq 0$ such that $E[u(\cdot, T)]<0$, then $u(x, t)$ is wave-like. 
Proof. Since $\phi(x) \in H_{c_{0}}^{1}(\mathbb{R})$, we have $u(x, T) \in H^{1}(\mathbb{R}) \cap H_{c_{0}}^{1}(\mathbb{R})$. For any small $\varepsilon>0$, when $E[u(x, T)]=-\varepsilon<0$, there exists $L>0$ such that

$$
\begin{aligned}
0 \leq & \frac{1}{2} \int_{L}^{\infty} e^{c_{0} x} u_{x}^{2}(x, T) d x<\frac{\varepsilon}{8}, \\
& -\frac{\varepsilon}{8}<\int_{L}^{\infty} e^{c_{0} x} V(u(x, T)) d x \leq 0 .
\end{aligned}
$$

Note that if we use smaller $c \geq 0$ instead of $c_{0}$ in the above inequalities, they still hold. And by the definition of $L$ we know that

$$
\int_{-L}^{L}\left(\frac{1}{2} u_{x}^{2}(x, T)+V(u(x, T))\right) d x<-\frac{3 \varepsilon}{4} .
$$

So we can find a sufficiently small $c>0$ such that $c<c_{0}$ and

$$
\int_{-L}^{L} e^{c x}\left(\frac{1}{2} u_{x}^{2}(x, T)+V(u(x, T))\right) d x<-\frac{\varepsilon}{2} .
$$

Then we have

$$
\Phi_{c}[u(\cdot, T)]=\int_{\mathbb{R}} e^{c x}\left(\frac{1}{2} u_{x}^{2}(x, T)+V(u(x, T))\right) d x<0 .
$$

So $u$ is wave-like.

In contrast to the bistable case, for monostable case boundedness of energy always implies extinction.

Lemma 4.3. Suppose that $E[u(\cdot, t)]$ is bounded from below for all $t \geq 1$, then $\lim _{t \rightarrow \infty} u(x, t)=0$ uniformly in $\mathbb{R}$.

Proof. Since the unique root of $V(u)$ is 0 , arguing as in Lemma 3.8 we know that $u(0, t) \rightarrow 0$ as $t \rightarrow \infty$. Then we prove this lemma by using Proposition 2.8 .

Lemma 4.4. Suppose that there exists $T \geq 0$ such that $E[u(\cdot, T)]<0$. Then $\lim _{t \rightarrow \infty} u(x, t)=1$ locally uniformly in $\mathbb{R}$.

Proof. The proof is similar to the proof of Lemma 3.4. If $E[u(\cdot, T)]<0$ for some $T \geq 0$, then there exists a sufficiently small $c>0$ such that $\Phi_{c}\left[\varphi_{L} u(\cdot, T)\right]<0$ for large enough $L>0$, where the cutoff function $\varphi_{L}$ is as in Lemma 3.4. Moreover, by the conditions (4.1) and (4.2), there exists $\delta_{0}>0$ defined by (2.9), such that Proposition 2.7 holds. Then we know that $R_{\delta_{0}}(t)>c t+R_{0}$ for some $R_{0} \in \mathbb{R}$. Similarly to Lemma 3.3, since the unique solution of Eq. (1.4) larger than $\delta_{0}$ is 1 in the whole of $\mathbb{R}$, we conclude that $\lim _{t \rightarrow \infty} u(x, t)=1$ locally uniformly in $\mathbb{R}$.

An immediate consequence of Lemmas 4.1 and 4.4 is the following.

Corollary 4.5. Suppose that $\lim _{t \rightarrow \infty} u(x, t)=0$ uniformly in $\mathbb{R}$, then $\lim _{t \rightarrow \infty} E[u(\cdot, t)]$ $=0$. 
We have thus established Theorems 4 and 5 .

Our last theorem in this section concerns with the threshold phenomena for monostable nonlinearities.

Theorem 6. Under the same conditions as in Theorem 4, suppose that (P1) through (P3) hold. Then one of the following holds:

1. $\lim _{t \rightarrow \infty} u_{\lambda}(x, t)=0$ uniformly in $x \in \mathbb{R}$ for every $\lambda>0$;

2. $\lim _{t \rightarrow \infty} u_{\lambda}(x, t)=1$ locally uniformly in $x \in \mathbb{R}$ for every $\lambda>0$;

3. There exists $\lambda^{*}>0$ such that

$$
\lim _{t \rightarrow \infty} u_{\lambda}(x, t)= \begin{cases}0, \text { uniformly in } x \in \mathbb{R}, & \text { for } 0<\lambda \leq \lambda^{*}, \\ 1, \text { locally uniformly in } x \in \mathbb{R}, & \text { for } \lambda>\lambda^{*} .\end{cases}
$$

Proof. Similarly to the proof of Theorem 3, if neither $\Sigma_{0}=\varnothing$ nor $\Sigma_{1}=\varnothing$, then $\Sigma_{1}$ is an open interval. The conclusion then follows.

Note that our sharp transition result above is nontrivial, e.g., for the generalized Fisher nonlinearity in (4.4) with $p>p_{c}$, where $p_{c}=3$ is the Fujita exponent (see, e.g., [20, Theorem 3.2]).

\section{Ignition Nonlinearity}

The ignition nonlinearity $f(u) \in C^{1}([0, \infty), \mathbb{R})$ satisfies

$$
f(u) \begin{cases}=0, & \text { in }\left[0, \theta_{0}\right] \cup\{1\}, \\ >0, & \text { in }\left(\theta_{0}, 1\right), \\ <0, & \text { in }(1, \infty),\end{cases}
$$

for some $\theta_{0} \in(0,1)$. We also suppose that there exists $\delta>0$ such that

$$
f(u) \text { is convex on }\left[\theta_{0}, \theta_{0}+\delta\right] \text {. }
$$

Under (1.3) and (5.1), except on the interval $\left[0, \theta_{0}\right]$, there exists at most one root $u=\theta^{\diamond}>1$ of $V(u)$. However, since $\limsup _{t \rightarrow \infty}\|u(x, t)\|_{L^{\infty}(\mathbb{R})} \leq 1$, without loss of generality, we suppose that $\|\phi\|_{L^{\infty}(\mathbb{R})}<\theta^{\diamond}$. So that we always have $V(u) \leq 0$.

Here are our main results concerning the long time behavior of solutions and their energy.

Theorem 7. Let $f$ satisfy conditions (5.1) and (5.2). Let $\phi(x)$ satisfy condition (1.3) and hypothesis (SD). Then one of the following holds.

1. $\lim _{t \rightarrow \infty} u(x, t)=1$ locally uniformly in $x \in \mathbb{R}$,

2. $\lim _{t \rightarrow \infty} u(x, t)=\theta_{0}$ locally uniformly in $x \in \mathbb{R}$,

3. $\lim _{t \rightarrow \infty} u(x, t)=0$ uniformly in $x \in \mathbb{R}$.

Theorem 8. Under the same assumptions as in Theorem 7, we have the following one-to-one relation.

1. $\lim _{t \rightarrow \infty} u(x, t)=1$ locally uniformly in $x \in \mathbb{R} \Leftrightarrow \lim _{t \rightarrow \infty} E[u(\cdot, t)]=-\infty$. 
2. $\lim _{t \rightarrow \infty} u(x, t)=\theta_{0}$ locally uniformly in $x \in \mathbb{R}$ or $\lim _{t \rightarrow \infty} u(x, t)=0$ uniformly in $x \in \mathbb{R} \Leftrightarrow \lim _{t \rightarrow \infty} E[u(\cdot, t)]=0$.

We prove the above theorems via a sequence of lemmas.

Lemma 5.1. Suppose that there exists $T \geq 0$ such that $E[u(\cdot, T)]<0$, then $\lim _{t \rightarrow \infty} u(x, t)=1$ locally uniformly in $\mathbb{R}$.

Proof. The arguments follow those in the proof of Lemma 3.4. If $E[u(\cdot, T)]<$ 0 for some $T \geq 0$, then there exists a sufficiently small $c>0$ such that $\Phi_{c}\left[\varphi_{L} u(\cdot, T)\right]<0$ for large enough $L>0$, where the cutoff function $\varphi_{L}$ is as in Lemma 3.4. Moreover, the condition in (5.1) implies that $\delta_{0}>\theta_{0}$, where $\delta_{0}$ is defined by (2.9). Then from Proposition 2.7, we know that $R_{\delta_{0}}(t)>c t+R_{0}$ for some $R_{0} \in \mathbb{R}$. Similarly to Lemma 3.3, since the unique solution of Eq. (1.4) larger than $\delta_{0}$ is 1 in the whole of $\mathbb{R}$, we conclude that $\lim _{t \rightarrow \infty} u(x, t)=1$ locally uniformly in $\mathbb{R}$.

Lemma 5.2. Suppose that $E[u(\cdot, t)]$ is bounded from below in $t$. Then either $\lim _{t \rightarrow \infty} u(x, t)=0$ uniformly in $\mathbb{R}$, or $\lim _{t \rightarrow \infty} u(x, t)=\theta_{0}$ locally uniformly in $\mathbb{R}$.

Proof. As in Lemma 3.8, there exists an unbounded increasing sequence $\left\{t_{n}\right\}$ such that

$$
\lim _{n \rightarrow \infty} V\left(u\left(0, t_{n}\right)\right)=0 .
$$

Since $\limsup \left\|u\left(\cdot, t_{n}\right)\right\|_{L^{\infty}(\mathbb{R})} \leq 1$, by the assumptions on the nonlinearity we have $V(u)=0$ if and only if $u \in\left[0, \theta_{0}\right]$. It implies that there exists a convergent subsequence of $\left\{u\left(0, t_{n}\right)\right\}$ (still denoted by $\left\{u\left(0, t_{n}\right)\right\}$ ) such that

$$
\lim _{n \rightarrow \infty} u\left(x, t_{n}\right)=\alpha,
$$

for some $\alpha \in\left[0, \theta_{0}\right]$.

Suppose that $0 \leq \alpha<\theta_{0}$, then there exists $T \geq 0$ sufficiently large such that $u(0, T)<\theta_{0}$. Then, for any $t>T, u(x, t) \equiv \theta_{0}$ is a supersolution of (1.1), so that $0 \leq u(x, t) \leq \theta_{0}$ uniformly in $\mathbb{R}$. From the definition of $f(u)$, it then follows that (1.1) becomes

$$
u_{t}(x, t)=u_{x x}(x, t),
$$

for any $t>T$. By [21, Proposition 48.4] we then have:

$$
\|u(\cdot, t)\|_{L^{\infty}(\mathbb{R})} \leq\left((4 \pi(t-T))^{-\frac{1}{4}}\|u(\cdot, T)\|_{L^{2}(\mathbb{R})} \rightarrow 0 \quad \text { as } t \rightarrow \infty .\right.
$$

It means that if $\alpha<\theta_{0}$, then $\alpha=0$, and $\lim _{t \rightarrow \infty} u(x, t)=0$ uniformly in $\mathbb{R}$.

If $\alpha=\theta_{0}$, then by using the same ODE argument as in the proof of Lemma 3.11, we can prove that $\lim _{t \rightarrow \infty} u(x, t)=\theta_{0}$ locally uniformly in $\mathbb{R}$. This proves the lemma.

Corollary 5.3. Suppose that $\lim _{t \rightarrow \infty} u(x, t)=1$ locally uniformly in $\mathbb{R}$, then $\lim _{t \rightarrow \infty} E[u(\cdot, t)]=-\infty$. 
Lemma 5.4. Both $\lim _{t \rightarrow \infty} u(x, t)=0$ uniformly in $\mathbb{R}$ and $\lim _{t \rightarrow \infty} u(x, t)=\theta_{0}$ locally uniformly in $\mathbb{R}$ imply $\lim _{t \rightarrow \infty} E[u(\cdot, t)]=0$.

Proof. By Lemma 5.1, $E[u(\cdot, t)] \geq 0$ for these behaviors. And since $V(u) \leq 0$ for any $u$, we have

$$
E[u(\cdot, t)] \leq \int_{\mathbb{R}} \frac{1}{2} u_{x}^{2}(x, t) d x
$$

So we only need to prove that the right-hand side of (5.7) converges to 0 as $t \rightarrow \infty$. From (SD), we have

$$
\int_{\mathbb{R}} \frac{1}{2} u_{x}^{2}(x, t) d x=\int_{0}^{\infty} u_{x}^{2}(x, t) d x \leq\left\|u_{x}(\cdot, t)\right\|_{L^{\infty}(\mathbb{R})} u(0, t) .
$$

We are done if $\lim _{t \rightarrow \infty} u(x, t)=0$ uniformly in $\mathbb{R}$, because $\left\|u_{x}(\cdot, t)\right\|_{L^{\infty}(\mathbb{R})}$ is bounded by standard parabolic regularity. So we only need to prove that $\left\|u_{x}(\cdot, t)\right\|_{L^{\infty}(\mathbb{R})} \rightarrow 0$ as $t \rightarrow \infty$ for the case $\lim _{t \rightarrow \infty} u(x, t)=\theta_{0}$ locally uniformly in $\mathbb{R}$.

We first prove that

$$
\lim _{t \rightarrow \infty} \sup _{|x| \leq R_{\theta_{0}}(t)}\left|u_{x}(x, t)\right|=0 .
$$

Otherwise there exist $\delta>0$, an increasing sequence $\left\{t_{n}\right\}$ with $\lim _{n \rightarrow \infty} t_{n}=\infty$, and a sequence $\left\{x_{n}\right\}$ with $\left|x_{n}\right| \leq R_{\theta_{0}}\left(t_{n}\right)$, such that $\left|u_{x}\left(x_{n}, t_{n}\right)\right| \geq \delta$. Since $\left|x_{n}\right| \leq R_{\theta_{0}}\left(t_{n}\right)$, for every $n$ we have $u\left(x_{n}, t_{n}\right) \geq \theta_{0}$, and without loss of generality we can assume that $x_{n}<0$ and $u_{x}\left(x_{n}, t_{n}\right)>0$. However, by standard parabolic regularity, there exists $M>0$, such that $\left|u_{x x}(x, t)\right|<M$ for all $x \in \mathbb{R}$ and all $t \geq 1$. This implies that for every $n$

$$
u\left(x_{n}+\frac{\delta}{M}, t_{n}\right)>\theta_{0}+\frac{\delta^{2}}{2 M}
$$

which contradicts our assumption that $\lim _{t \rightarrow \infty} u(x, t)=\theta_{0}$ locally uniformly in $\mathbb{R}$.

Then we estimate $\left|u_{x}(x, t)\right|$ on $\left(R_{\theta_{0}}(t), \infty\right)$. Multiplying $(1.1)$ by $u_{x}$ and integrating from the leading edge $R_{\theta}(t)$ to $\infty$, which is justified by Proposition 2.1 , for any $\theta \in\left(0, \theta_{0}\right]$, we have

$$
\int_{R_{\theta}(t)}^{\infty} u_{x}(x, t) u_{t}(x, t) d x=\int_{R_{\theta}(t)}^{\infty} u_{x}(x, t) u_{x x}(x, t) d x
$$


since $f(u)=0$ for any $u \in\left[0, \theta_{0}\right]$. Integrating by parts and applying CauchySchwarz inequality, we obtain

$$
\begin{aligned}
\frac{1}{2} u_{x}^{2}\left(R_{\theta}(t), t\right) & \leq\left(\int_{R_{\theta}(t)}^{\infty} u_{x}^{2}(x, t) d x \int_{R_{\theta}(t)}^{\infty} u_{t}^{2}(x, t) d x\right)^{\frac{1}{2}} \\
& \leq\left(\theta \max _{x \in \mathbb{R}}\left|u_{x}(x, t)\right| \int_{R_{\theta}(t)}^{\infty} u_{t}^{2}(x, t) d x\right)^{\frac{1}{2}} \\
& \leq\left(\theta_{0} \max _{x \in \mathbb{R}}\left|u_{x}(x, t)\right| \int_{\mathbb{R}} u_{t}^{2}(x, t) d x\right)^{\frac{1}{2}} .
\end{aligned}
$$

Since $E[u(\cdot, t)]$ is bounded from below in $t$, there exists an increasing sequence $\left\{t_{n}\right\}$ such that $\lim _{n \rightarrow \infty} t_{n}=\infty$ and

$$
\lim _{n \rightarrow \infty} \int_{\mathbb{R}} u_{t}^{2}\left(x, t_{n}\right) d x=0
$$

In turn, since $\theta$ is arbitrary in $\left(0, \theta_{0}\right]$, we have

$$
\lim _{n \rightarrow \infty} \sup _{x>R_{\theta_{0}}\left(t_{n}\right)}\left|u_{x}\left(x, t_{n}\right)\right|=0 .
$$

This means that the right-hand side of (5.7) converges to 0 on the sequence $\left\{t_{n}\right\}$. The statement of the lemma then follows, since $E[u(\cdot, t)]$ is non-increasing.

We have now proved our convergence and equivalence theorems for the ignition nonlinearity. Studying the threshold phenomena for ignition nonlinearity is a little different from the situation with bistable nonlinearity. The main difficulty is to show that the threshold set contains only a single point, since we cannot construct the type of barrier used in the proof of Theorem 3. Instead we modify the proof by Zlatoš in [10], which uses a rescaling technique for dealing only with the initial condition in the form of a characteristic function.

Lemma 5.5. Let $f:[0, \infty) \rightarrow \mathbb{R}$ be a Lipschitz function with $f(0)=0$. Let $U(x, t): \mathbb{R} \times[0, \infty) \rightarrow[0, \infty)$ be a classical solution of

$$
U_{t}=U_{x x}+f(U),
$$

which is uniformly continuous up to $t=0$. Denote by $U_{1}(x, t)$ and $U_{2}(x, t)$ the solutions of Eq. (5.15) with initial conditions $U_{1}(x, 0)$ and $U_{2}(x, 0)$, respectively, and assume $0 \leq U_{1}(x, 0) \leq U_{2}(x, 0)$ for any $x \in \mathbb{R}$, and $U_{1}\left(x_{0}, 0\right)<$ $U_{2}\left(x_{0}, 0\right)$ for some $x_{0} \in \mathbb{R}$. Assume also that for any $\rho>0$ the set $\Omega_{0, \rho}=\{x \in$ $\left.\mathbb{R}: U_{2}(x, 0) \geq \rho\right\}$ is compact. Finally, assume that there are $0<\theta_{1}<\theta_{2}$ and $\varepsilon_{1}>0$ such that for any $\theta \in\left[\theta_{1}, \theta_{2}\right]$ and $\varepsilon \in\left[0, \varepsilon_{1}\right]$, we have

$$
f\left(\theta+\varepsilon\left(\theta-\theta_{1}\right)\right) \geq(1+\varepsilon) f(\theta),
$$

and assume $\left\|U_{1}\right\|_{L^{\infty}(\mathbb{R} \times(0, \infty))}<\theta_{2}$ for any $t \in[0, \infty)$. Then

$$
\liminf _{t \rightarrow \infty} \inf _{U_{1}(x, t)>\theta_{1}} \frac{U_{2}(x, t)-\theta_{1}}{U_{1}(x, t)-\theta_{1}}>1,
$$


with the convention that the infimum over an empty set is $\infty$.

Proof. It is essentially [10, Lemma 4].

Theorem 9. Under the same conditions as in Theorem 7, suppose that (P1) through (P3) hold. Then one of the following holds:

1. $\lim _{t \rightarrow \infty} u_{\lambda}(x, t)=0$ uniformly in $x \in \mathbb{R}$ for every $\lambda>0$;

2. There exists $\lambda^{*}>0$ such that

$$
\lim _{t \rightarrow \infty} u_{\lambda}(x, t)= \begin{cases}0, \text { uniformly in } x \in \mathbb{R}, & \text { for } 0<\lambda<\lambda^{*}, \\ \theta_{0}, \text { locally uniformly in } x \in \mathbb{R}, & \text { for } \lambda=\lambda^{*}, \\ 1, \text { locally uniformly in } x \in \mathbb{R}, & \text { for } \lambda>\lambda^{*} .\end{cases}
$$

Proof. Similarly to the proof of Theorem 3, we can show that if $\Sigma_{0} \neq(0, \infty)$, then both $\Sigma_{0}$ and $\Sigma_{1}$ are open intervals, and hence $\mathbb{R}_{+} \backslash\left(\Sigma_{0} \cup \Sigma_{1}\right)$ is a closed interval. Then we only need to prove that $\mathbb{R}_{+} \backslash\left(\Sigma_{0} \cup \Sigma_{1}\right)$ contains only a single point. We need to verify that if $f(u)$ satisfies (5.1) and (5.2), then there exists $\varepsilon_{1}>0$, and $0<\theta_{1}<\theta_{0}<\theta_{2}<1$ such that condition (5.16) holds. Note that convexity of $f(u)$ on $\left[\theta_{0}, \theta_{0}+\delta\right]$ implies that $f(u)$ is nondecreasing on $\left[\theta_{0}, \theta_{0}+\delta\right]$, and $\theta_{0}+\delta<1$. Taking $\varepsilon_{1}=\delta / 2, \theta_{1}=\theta_{0} / 2, \theta_{2}=\left(3 \theta_{0}+\delta\right) / 3$, we only need to prove that (5.16) holds for any $\varepsilon \in\left[0, \varepsilon_{1}\right]$ and $\theta \in\left[\theta_{0}, \theta_{2}\right]$. Let $\alpha:=\theta-\theta_{0} \in[0, \delta / 3]$. We have the following estimate of the left-hand side of $(5.16)$,

$$
f\left(\theta+\varepsilon\left(\theta-\theta_{1}\right)\right)=f\left(\theta_{0}+(1+\varepsilon) \alpha+\frac{\varepsilon \theta_{0}}{2}\right) \geq f\left(\theta_{0}+(1+\varepsilon) \alpha\right),
$$

since $\theta+\varepsilon\left(\theta-\theta_{1}\right)<\theta_{0}+\delta$. By convexity we also have

$$
f\left(\theta_{0}+\alpha\right) \leq \frac{f\left(\theta_{0}+(1+\varepsilon) \alpha\right)}{1+\varepsilon}+\frac{\varepsilon f\left(\theta_{0}\right)}{1+\varepsilon},
$$

which proves

$$
f\left(\theta_{0}+(1+\varepsilon) \alpha\right) \geq(1+\varepsilon) f\left(\theta_{0}+\alpha\right),
$$

for any $\varepsilon \in\left[0, \varepsilon_{1}\right]$ and $\alpha \in\left[0, \theta_{2}-\theta_{0}\right]$. Hence (5.16) holds for any $\theta \in\left[\theta_{1}, \theta_{2}\right]$ and $\varepsilon \in\left[0, \varepsilon_{1}\right]$.

Then we suppose that there exist two distinct values $0<\lambda_{1}<\lambda_{2}$ in the threshold set $\mathbb{R}_{+} \backslash\left(\Sigma_{0} \cup \Sigma_{1}\right)$. Denote by $u_{\lambda_{1}}(x, t)$ and $u_{\lambda_{2}}(x, t)$ these solutions with initial conditions $\phi_{\lambda_{1}}$ and $\phi_{\lambda_{2}}$, respectively. Taking $\theta_{1}, \theta_{2}$ as above, there exists $T>0$ such that $\left\|u_{\lambda_{1}}\right\|_{L^{\infty}(\mathbb{R} \times(0, \infty))}<\theta_{2}$, for any $t \geq T$. And for any $t \geq T$, let $U_{1}(x, t):=u_{\lambda_{1}}(x, t-T)$ and $U_{2}(x, t):=u_{\lambda_{2}}(x, t-T)$. Obviously all the assumptions of Lemma 5.5 hold. So there exists $r>1$ such that

$$
\liminf _{t \rightarrow \infty} \frac{U_{1}(0, t)-\theta_{1}}{U_{2}(0, t)-\theta_{1}} \geq r
$$

But both $U_{1}(0, t)$ and $U_{2}(0, t)$ converge to $\theta_{0}$ as $t \rightarrow \infty$. So that the left-hand side of (5.21) must be 1 , which is a contradiction.

Remark 5.6. The $C^{1}$ property of $f(u)$ and condition (5.1) imply that $f^{\prime}\left(\theta_{0}\right)=$ 0 . If we suppose that $f(u) \in C[0, \infty) \cap C^{1}\left(\theta_{0}, \infty\right)$, together with (5.1), and 
$\lim _{u \rightarrow \theta_{0}^{+}} f^{\prime}(u)>0$, then without local convexity condition (5.2) all the conclusions about convergence, equivalence, and sharp transition in this section still hold.

\section{Acknowledgments}

The authors are grateful to P. Gordon, H. Matano, V. Moroz and M. Novaga for valuable discussions.

\section{References}

[1] Zeldovich, Y.B., Frank-Kamenetski, D.A.: On the theory of uniform flame propagation. Dokl. Akad. Nauk SSSR 19, 693-697 (1938)

[2] Zeldovich, Y.B., Barenblatt, G.I., Librovich, V.B., Makhviladze, G.M.: The Mathematical Theory of Combustion and Explosions. Consultants Bureau, New York (1985)

[3] Buckmaster, J.D., Ludford, G.S.S.: Lectures on Mathematical Combustion. Society for Industrial and Applied Mathematics, Philadelphia (1983)

[4] Merzhanov, A.G., Rumanov, E.N.: Physics of reaction waves. Rev. Mod. Phys. 71, 1173-1210 (1999)

[5] Murray, J.D.: Mathematical Biology. Springer, Berlin (1989)

[6] Mikhailov, A.S.: Foundations of Synergetics. Springer, Berlin (1990)

[7] Kerner, B.S., Osipov, V.V.: Autosolitons. Kluwer, Dordrecht (1994)

[8] Kanel', J.I.: Stabilization of the solutions of the equations of combustion theory with finite initial functions. Mat. Sb. (N.S.) 65, 398-413 (1964)

[9] Aronson, D.G., Weinberger, H.F.: Nonlinear diffusion in population genetics, combustion and nerve pulse propagation. In: Partial Differential Equations and Related Topics. Lecture Notes in Mathematics, vol.446, pp. 5-49. Springer, Berlin (1975)

[10] Zlatoš, A.: Sharp transition between extinction and propagation of reaction. J. Am. Math. Soc. 19, 251-263 (2006)

[11] Feireisl, E., Petzeltová, H.: Convergence to a ground state as a threshold phenomenon in nonlinear parabolic equations. Differ. Integral Equ. 10, 181196 (1997)

[12] Flores, G.: The stable manifold of the standing wave of the Nagumo equation. J. Diff. Equ. 80, 306-314 (1989)

[13] Du, Y., Matano, H.: Convergence and sharp thresholds for propagation in nonlinear diffusion problems. J. Eur. Math. Soc. 12, 279-312 (2010) 
[14] Poláčik, P.: Threshold solutions and sharp transitions for nonautonomous parabolic equations on $\mathbb{R}^{n}$. Arch. Ration. Mech. Anal. 199, 69-97 (2011)

[15] Matano, H.: Private communication (2010)

[16] Busca, J., Jendoubi, M.A., Poláčik, P.: Convergence to equilibrium for semilinear parabolic problems in $\mathbb{R}^{n}$. Comm. Partial Differ. Equ. 27, 1793-1814 (2002)

[17] Fašangová, E., Feireisl, E.: The long-time behaviour of solutions to parabolic problems on unbounded intervals: the inuence of boundary conditions. Proc. R. Soc. Edinb. Sect. A 129, 319-329 (1999)

[18] Cortázar, C., del Pino, M., Elguetal, M.: The problem of uniqueness of the limit in a semilinear heat equation. Comm. Partial Differ. Equ. 24, 2147-2172 (1999)

[19] Feireisl, E.: On the long time behaviour of solutions to nonlinear diffusion equations on $\mathbf{R}^{n}$. NoDEA Nonlinear Differ. Equ. Appl. 4, 43-60 (1997)

[20] Aronson, D.G., Weinberger, H.F.: Multidimensional nonlinear diffusion arising in population genetics. Adv. Math. 30, 33-76 (1978)

[21] Quittner, P., Souplet, P.: Superlinear parabolic problems. Birkhäuser Advanced Texts: Basler Lehrbücher. Birkhäuser Verlag, Basel (2007)

[22] Friedman, A.: Partial Differential Equations of Parabolic Type. Prentice-Hall, Inc., Englewood Cliffs (1964)

[23] Fife, P.C., McLeod, J.M.: The approach of solutions of nonlinear diffusion equations to travelling front solutions. Arch. Ration. Mech. Anal. 65, 335-361 (1977)

[24] Evans, L.C.: Partial Differential Equations. Graduate Studies in Mathematics, 19. American Mathematical Society, Providence (1998)

[25] Muratov, C.B., Novaga, M.: Global exponential convergence to variational traveling waves in cylinders. SIAM J. Math. Anal. 44, 293-315 (2012)

[26] Lunardi, A.: Analytic semigroups and optimal regularity in parabolic problems. Volume 16 of Progress in Nonlinear Differential Equations and their Applications. Birkhäuser, Basel (1995)

[27] Muratov, C.B.: A global variational structure and propagation of disturbances in reaction-diffusion systems of gradient type. Discret. Contin. Dyn. Syst. Ser. B 4, 867-892 (2004)

[28] Muratov, C.B., Novaga, M.: Front propagation in infinite cylinders. I. A variational approach. Comm. Math. Sci 6, 799-826 (2008)

[29] Lucia, M., Muratov, C.B., Novaga, M.: Existence of traveling waves of invasion for Ginzburg-Landau-type problems in infinite cylinders. Arch. Rational Mech. Anal. 188, 475-508 (2008)

[30] Berestycki, H., Lions, P.L.: Nonlinear scalar field equations. I. Existence of a ground state. Arch. Rational Mech. Anal. 82, 313-345 (1983) 
[31] Fife, P.C.: Long time behavior of solutions of bistable nonlinear diffusion equations. Arch. Ration. Mech. Anal. 70, 31-46 (1979)

[32] Lieb, E.H., Loss, M.: Analysis. Am. Math. Soc. (2001)

C. B. Muratov

Department of Mathematical Sciences

New Jersey Institute of Technology

Newark

NJ 07102

USA

e-mail: muratov@njit.edu

$\mathrm{X}$. Zhong: The author is deceased.

Received: 4 September 2012.

Accepted: 5 January 2013. 